\title{
Individual heterogeneity and its importance for metapopulation dynamics.
}

\author{
Stefano Masier ${ }^{* 1}$, Maxime Dahirel ${ }^{2}$, Frederik Mortier ${ }^{1} \&$ Dries Bonte $^{1}$
}

1: Ghent University, Dept. Biology, Terrestrial Ecology Unit, K.L. Ledeganckstraat 35, 9000 Ghent, Belgium.

2: Université Côte d'Azur, INRAE, CNRS, ISA, 400 route des Chappes, 06903 Sophia Antipolis, France.

* Corresponding Author: Stefano Masier.

Email: stefano.masier.89@gmail.com 


\begin{abstract}
Habitat fragmentation reduces the area of habitat patches and their connectedness in the landscape. The level of connectedness impacts connectivity and metapopulation dynamics, and is therefore a central tenet in conservation biology. Connectedness equally generates sorting of phenotypes within the spatial network and therefore impacts local and regional eco-evolutionary dynamics. Paradoxically, recommendations for species conservation rely on principles derived from theory that neglects any individual phenotypic heterogenety and spatial organization.

By creating experimental metapopulations using the model species Tetranychus urticae (twospotted spider mite) with three levels of landscape connectedness and by regularly removing phenotypic structure in a subset of these populations, we tested the degree to which regional and local population dynamics are determined both by network connectedness and the phenotypic spatial organization.

Our results show that some aspects of metapopulation dynamics can be attributed to the evolution of dispersal. More importantly, we find self-organization of phenotypic spatial structure to equalize the effects of reduced connectedness on metapopulation dynamics. These changes were all in the direction of improved metapopulation persistence. Contrary to expectations, the most connected local patches showed an overall reduced local population size, possibly originating from a faster depletion of resources from immigrants or transiting individuals.

This experiment shows how metapopulation dynamics can significantly deviate from theoretical expectations if individual heterogeneity is considered, and more importantly that disruption of this phenotypic self-structuring may drive metapopulation dynamics towards higher risks of extinction.
\end{abstract}




\section{Introduction}

Habitat fragmentation has a deep impact on the population structure and demography of almost every species at any trophic level [1]-[4]. The physical disruption of a previously continuous habitat into a series of patches separated by a more hostile matrix reduces the amount of habitat and its connectivity. Connectivity describes the realized gene flow through individual or propagule dispersal among patches of suitable habitat. It therefore depends on the connectedness of the patches, defined as their spatial configuration and the resulting inter patch distances [5]-[7]. Connectivity also depends on the dispersal capacities of the species involved [8]. Connectedness loss decreases connectivity, and therefore the probability that an empty patch will be colonized, or that patches at the brink of extinction will be rescued. Overall, it tends to lower the persistence and stability of a metapopulation [9], [10] and is therefore of prime importance for conservation biology.

Connectedness impacts local and regional fluctuations in population sizes, as well as the synchronicity of these changes within the entire metapopulation [11]. Theory predicts that lower effective dispersal between disconnected patches raises both local and regional variabilities in population size and causes the whole metapopulation to be more vulnerable to local extinctions [12], [13] even if the local characteristics of the system (i.e. resources availability) are kept constant. Alternatively, a more connected network will show a higher level of spatial synchronicity [12], [14], which makes the whole system also more vulnerable to global extinction as there is no rescuing potential when all local populations decrease at the same time [15], [16]. Connectivity can thus be considered a double-edged sword for metapopulation conservation [17].

These theoretical developments have, however, mostly ignored evolutionary dynamics and the potential for eco-evolutionary feedbacks. Landscape connectedness directly affects the costs of dispersal [18], imposing selection pressures on dispersal and life history. Subsequent evolution will in turn feedback on connectivity and metapopulation demography [19], [20]. These ecoevolutionary dynamics involve changes in relatedness and phenotypic structure within metapopulations. An increased spatial genetic structure resulting from low levels of gene flow will elevate competitive interactions among kin, which is known to be an important driver of dispersal [21], thus potentially imposing negative feedbacks on the metapopulation dynamics [22]. Because dispersal is performed by a non-random subset of individuals from the population that share specific life history traits [18], [23], [24] and physiology [25], the phenotypic (kind) structure [26] will be altered by changes in connectedness. This has the potential to affect population dynamics through impacts on population growth. 
Metapopulation dynamics are consequently expected to be influenced by the joint action of localand metapopulation-level selection [27], and will lead to a self-organisation of the distribution of phenotypes in the spatial network. The position and local connectedness of each patch within the spatial network will determine both the number and phenotype of the immigrants, shaping local demography [8], [28]-[30]. Patches with higher levels of local connectedness are for instance expected to have an increased immigration, that should theoretically increase population densities [27], [31]. Alternatively, as less connected patches will receive lower numbers of immigrants, they will become genetically more isolated, kin-structured and inbred. Besides the direct impacts on performance from potential inbreeding depression [32], emigration may vary plastically in order to avoid inbreeding [33], [34]. All in all, because local eco-evolutionary feedbacks have the potential to impact connectivity of each patch in the network, they may control local and regional stability, and therefore metapopulation viability [10].

Species' responses to connectedness loss are mostly attributed to purely ecological causes [35], or dispersal evolution [36]. It is however obvious that we need quantitative and qualitative insights on how eco-evolutionary dynamics affect both regional and local population dynamics when connectedness is altered. We demonstrated earlier that changes in connectedness impose genetic and epigenetic changes in dispersal timing, dispersal costs and fecundity [27] in experimental metapopulations of two-spotted spider mites Tetranychus urticae. We here show by means of an individual based model that metapopulation dynamics are better predicted by the evolutionary divergence in dispersal across different landscape contexts as earlier reported [27] rather than by dispersal costs from connectedness loss per se. Next, and more importantly, we demonstrate experimentally that the spatial self-organization of phenotypes (i.e., the emerging kin and kind structure) within a metapopulation is equalizing metapopulation dynamics across these connectedness levels. We initialized replicated experimental metapopulations according to three levels of "regional" connectedness, and implemented a randomization treatment in which the genoand phenotypic structure was destroyed while leaving demographic attributes (densities, stage, sex and age-structure) untouched. This treatment thus removes the emerging spatial kin and kind structure [27]. We quantified and compared average local and metapopulation sizes, as well as the level of demographic fluctuations (alpha or local and gamma or metapopulation variability) and synchrony in local population size changes (beta variability) between treatments [11]. As variation in local connectedness may also affects local dynamics, we additionally isolated and compared responses from patches within the same spatial network varying in their degree of local connectedness. 


\section{Results}

\section{Dispersal versus connectedness and metapopulation dynamics}

A theoretical metapopulation model reflecting population dynamics in our experimental system shows local $(\alpha)$ and regional $(\gamma)$ variabilities are expected to increase with higher dispersal mortality under connectedness loss, but that $(\beta)$ synchrony remains unaffected. Local and regional variability decrease theoretically as well, while synchrony increases, when emigration rates decrease by evolution (fig. 1). No effects on equilibrium densities were found. Only observed patterns in variability relative to earlier quantified evolved dispersal rates match those predicted from the model. Heterogeneity in these dynamics across treatments is thus better explained by the average evolutionary trait divergence rather than ecological costs of connectivity loss.

\section{Individual heterogeneity and metapopulation dynamics}

In absence of randomization, metapopulation size was directly linked to connectedness, with more loosely connected landscapes reaching higher metapopulation sizes (fig. 2.1A). The implemented connectedness treatments did not induce changes in local and regional variability or synchrony (see fig. 2.1). However, when both kin and kind structure were removed by the randomization treatment, different effects on metapopulation size emerged depending on the level of global connectedness (fig. 2.2). Relative to the non-shuffled replicates, metapopulation size increased in the least connected, but decreased in the most connected randomized metapopulations. The randomization treatment equally induced a larger local and metapopulation variability in the least connected metapopulations but no changes in synchrony ( $\beta$ variability) were detected. Effect sizes of the randomization treatment are summarized in fig. 3 .

\section{Individual heterogeneity and local dynamics}

Independently of the landscape level of connectedness, local population size was negatively linked with local connectedness: the most connected (central) patches in networks showed on average the lowest population size, followed by edge and then finally corner patches (fig.4). These changes in density were most pronounced under the randomization treatment. Local $(\alpha)$ variabilities did not change according to the local level of connectedness. 


\section{Discussion}

Metapopulation dynamics, as synthesized by average population sizes, population size variability and synchrony are known to be impacted by the level of connectedness [11], [13], [37], dispersal [38], [39] and the interaction of dispersal with local environmental and demographic variation [40]. We quantified these dynamics in series of simulated and experimental metapopulations, and found variation in evolved dispersal rather than costs across metapopulations to match predictions on population size variability. Dispersal in general is phenotype-dependent [41], [42], leading to sorting of phenotypes in metapopulations [23], [43]. We provide experimental evidence that such a phenotypic self-organization is an additional and powerful driver of metapopulation dynamics.

Although landscapes shared the same amount of resources and thus had theoretically the same carrying capacity, the least connected metapopulations reached higher average population sizes. Lower local population sizes in metapopulations are typically attributed to genetic load when dispersal is constrained [44], [45]. In the present study, however, these changes cannot be attributed to evolved population growth or dispersal rates, as they were similar across the three connectedness treatments [27]. Starvation resistance evolved in the least connected metapopulations [27] as a putative adaptation to an increased exposure to environments without resources in between the patches. As such an adaptation may strengthen positive $\mathrm{r}-\mathrm{K}$ correlations [46], [47], it provides a tentative explanation for the higher average population sizes in the least connected metapopulation. An increased stress resistance should not only increase survival during transfer, but equally allow increased survival when densities are overshooting 'natural' carrying capacities. Putting it another way, under temporal environmental variation, stress evolution increases performance of populations, as long as the mean stress is far enough from the upper limit of the ecological niche [48]. Resource-consumer dynamics combined with differences in $\mathrm{r}-\mathrm{K}$ covariation may drastically affect carrying capacities as predicted from simple (Lotka-Volterra) population models [49]; we here extend this view by including the potential for local adaptation to altered resource conditions.

Theory predicts that lower connectedness and dispersal intensify local and regional variabilities in population sizes [50], and increase spatial asynchrony within the metapopulation [13]. In our experiment, the similar levels of local population $(\alpha)$ variability, metapopulation-level $(\gamma)$ variability and asynchrony ( $\beta$ variability) across the imposed (non-randomized) connectivity treatments suggest that that the differences in level of connectedness do not impose sufficiently strong parallel selection on dispersal or the dispersal costs (see also Supplementary material S1). Contrasting the model outcomes to the evolved dispersal rates across the experimental replicates indeed shows that data and model outcomes correlate especially for local and regional variability well, while 
opposite patterns are to be expected when contrasting only dispersal costs in relation to connectedness (fig.1). As multiple traits evolve when connectedness is changing, including some at the local level [27], [29], they may balance each other and erode systematic demographic changes across the connectedness treatments.

General metapopulation theory does not take into account that any local variation in dispersal and demography is promoting phenotypic and genotypic sorting in the network, and thereby a spatial structuring in traits and relatedness. It is, however, known that spatial heterogeneity in demographic rates may alter and even reverse population-level predictions from mean-field theory [51]-[53] Such variation is typically assumed to be environmentally driven, but local phenotypic structuring may be at the basis of such spatial variation as well [27]. Our reshuffling treatment, where population densities and stage structure were maintained but not genetic (kin) and phenotypic (kind) structure [26] provided a unique opportunity to study the population demographic consequences of this selforganizing mechanism in metapopulations. Under this reshuffling treatment, differences in average population sizes increased, with differences up to $25 \%$ between the least connected and the two more connected experimental metapopulations. Local $(\alpha)$ and regional $(\gamma)$ variability decreased in the two most connected metapopulation treatments as well, while asynchrony remained stable. This shows that (meta-)population variability shifts into the directions anticipated from ecological theory, including our simulation model, when spatial individual heterogeneity is removed. The widely accepted theoretical paradigm on the role of connectivity for metapopulation dynamics is thus falsified when individual heterogeneity is removed [54]-[57].

When kin and kind structure is removed by reshuffling, the least connected metapopulations show higher levels of variability. This accords with predictions from the loss of connectivity [58]. As kincompetition is systematically removed by this treatment, overall dispersal rates can be anticipated to have dropped [21], either plastically as a conditional response or evolutionary as an adaptation to the absence of any kin structure during the experiment. No support for such evolved changes was found earlier [27]. As we expect the reshuffling treatment to destroy kin structure equally among all connectedness treatments, a reduced dispersal conditional to low levels of relatedness [33] should be similar across the three levels of connectedness. We can therefore only speculate that delayed dispersal in the least connected metapopulations [27] together with an overall reduction due to the breakdown of kin structure may have brought the level of connectivity beyond a threshold value that eventually translated into these diverging local dynamics. However, as synchrony was equal among all reshuffling and connectedness treatments, such connectivity changes are likely not pronounced. 
Randomization also improved global population size in the least connected setups, while it had a negative effect in the others. Higher population sizes can arise from increased group benefits [59][61], but as for kin-competition, we anticipate the reshuffling to equally remove any kin-benefits of group living among the connectedness treatments. A putative explanation would thus involve the balance between the loss of said group-living benefits and the spread of favourable genotypes without having to incur into the costs of dispersal. When such costs are high, the balance would be positive and randomization would be expected to yield a positive effect on metapopulation size; otherwise, the total effect can be expected to be negative.

Our experiments additionally show how differences in connectedness within the networks affect local population sizes. As the central patches showed the highest level of connectedness in all metapopulations, increased population sizes due to positive immigration-emigration balances were expected [27]. Surprisingly, opposite patterns were found with central patches systematically showing lower densities. This finding suggests the existence of local demographic changes that are not captured by the metapopulation-level rates of dispersal. We again can only speculate about the exact mechanisms leading to these paradoxical results. On the one hand, the earlier observed (intergenerationally plastic) lower reproductive rates [27] may have 'evolved' as a response to potential overshooting from increased immigration [62], [63]. On the other hand, as we did not record the total number of individuals, but only the adult females, it is similarly possible that juvenile development was slowed down due to resource limitation. Is is known that $T$. urticae exhibits longer development times when faced with suboptimal food sources, while longevity and egg-laying performances are not affected [64]; in our setup, this likely caused a reduced number of adults during the counts of central patches relative to the others.

Theory predicts that populations inhabiting more fragmented landscapes should be less stable, with a higher chance of both local and regional extinctions [10], [65]. It is also known that dispersal can counteract this through spatial averaging or rescue effects [66]-[68]. While the exact processes underlying demographic shifts under the implemented reshuffling treatment remain speculative, we demonstrate the importance of phenotypic and genotypic self-organization for metapopulation dynamics. Since most of the available theory neglect individual variability, such self-organizing properties could not be anticipated. Importantly, in those setups where connectedness was at the lowest, spatial self-organization eroded fluctuations in population sizes, thereby facilitating potential rescue. These insights are extremely relevant for conservation, as the translocation of individuals is here shown to add an unrecognised risk [69] to the persistence of the metapopulation.

While phenotypic variation has been shown to explain patterns in biodiversity [23], [70] we here demonstrate its importance for metapopulation demography, and in extension metapopulation 
conservation. Our reshuffling treatment can be considered as an extreme case of hypermobility within the spatial network. Our experiment also pinpoints the consequences of such a hypermobility from an applied perspective [71]-[73]. Apart from the potential to impose maladaptation, or to hinder adaptation [74], [75], hypermobility is also able to lead to changes in the local and regional population dynamics, even to such a degree that local population extinction chances increase.

\section{Materials and Methods}

\section{Individual-based model}

We generated predictions on the role of connectivity in metapopulation variability ( $\alpha, y$ and $\beta$ ) with an individual-based metapopulation model. We expected a direct role of the connectedness on metapopulation dynamics. Having observed dispersal timing evolution [27], we also expected this indirectrole of connectedness on metapopulation dynamics. We simulated the direct and indirect effect of connectedness on metapopulation variabilities to contrast with the observed dynamics in the experiment, in order to estimate the relative impact of both.

We simulated metapopulations, resembling our experimental systems of $3 \times 3$ connected patch networks, in Python (see "Data and code accessibility"). We constructed an individual-based version of the classic metapopulation model in discrete generations [76]. Individuals did not differ in traits within a simulation and, thus, only simulated dispersal and demographic dynamics. Ongoing evolution is completely excluded. In a first set of simulations, we analyzed different levels of dispersal mortality to simulate higher dispersal costs in order to analyze the direct effect of a lower connectedness on metapopulation dynamics. We decided to use mortality as a superparameter that encompasses both the direct (i.e. death during dispersal) and the deferred (i.e. reduced reproductive success due to energy expenditure) costs of the dispersal event [18].

In a second set of simulations, we implemented variation in dispersal propensity to analyze the effect of dispersal timing on metapopulation dynamics. In our experiment, an on average earlier dispersal timing evolved with higher connectedness [27]. This would have more individuals dispersed before new resources are added to each patch. As in presence of abundant pro-capita resources the probability of emigration is known to fall sharply [33], [77], [78], dispersal timing correlates highly with dispersal frequency. .

A detailed description of the model is provided in supplementary materials (SI2).

\section{Study species and experiment description}

To perform the experiment we chose the haplodiploid spider mite Tetranychus urticae Koch 1836 (Acari: Tetranychidae) ("two-spotted spider mite"). 
We studied the ecological dynamics of spider mites in 24 independent experimental metapopulations, sorted in three connectedness levels and one randomization/control treatment (see below). All experimental metapopulations consisted of a grid of 9 patches, which organized a Moore neighboorhood, with each patch a square of $5 \times 5 \mathrm{~cm}^{2}$ cut from one of the two main leaves of a two-weeks-old bean plant. Patches were connected through Parafilm bridges, creating a 3-by-3 grid; each bridge was about $0.5 \mathrm{~cm}$ wide. The length of the plastic bridges between patches in the same row/column was defined according to the connectivity treatment: closely connected $(4 \mathrm{~cm}$ length), averagely connected $(8 \mathrm{~cm})$ and loosely connected $(16 \mathrm{~cm})$. Diagonal bridges to the central patches were longer due to triangularity. As such, each treatment consisted of 9 patches, with different levels of local connectedness. Central patches were connected to all other patches (8 links), while side and corner patches were less connected (5 and 3 links, respectively, see [27], in particular figure 1 for a more detailed description of the experimental setup). Each connectedness treatment was 8 times replicated.

Further details on the experimental setup are provided as supplementary material (SI3).

Even if every patch should theoretically present the same conditions, local variation in population densities, connectedness and phenotypic/genotypic structure could nonetheless arise and influence local dynamics. To tease out the effect of phenotypic and genotypic heterogeneity (kin/kind structure) from the rest, we selected three landscapes out of eight from each connectedness level to undergo a randomization treatment. Every week, all the adult females from each of these metapopulations were collected using a thin paintbrush, put on a fresh bean leaf to reduce stress as much as possible and then moved back on a random patch in the same setup, maintaining the absolute numbers for each patch but reshuffling the individuals. This was done to destroy kin and kind structures (genetic relatedness and phenotypic similarity; [26]) as well as any local adaptation caused i.e. by local densities, while maintaining the ecological effect of landscape fragmentation on population densities. To ensure any effect observed was not merely due to the stress of the translocation, females from the non-randomized treatments were also picked up and moved to a temporary leaf; they were however not reshuffled and simply put back on the patch they were taken from after a short time.

Each patch was initialized at the start of the experiment using 5 adult females and 2 adult males taken randomly from our stock population (63 individuals per metapopulation, 1512 individuals overall) to ensure equal starting conditions and a sufficient genetic variability [26]. After a 15-dayslong ( 1.5 generations) "burn-in" period during which no count or reshuffling was performed, the metapopulations were monitored for six months ( 18 generations at the described conditions).

\section{Data analysis}


A complete and formal description of the Bayesian multivariate multilevel/mixed model is included within the model code (see "Data and code availability") and in supplementary material (SI4).

Our model contained nine submodels, one per patch in an experimental metapopulation (identified by their coordinate in the patch network). For each patch, we assumed weekly population counts were Poisson distributed, and depended on treatment (fixed effect; interaction between global connectedness and randomization) as well as on the identity of the experimental metapopulation (random intercept). The replicate identity random effects for all nine submodels were included in a common variance-covariance matrix to account for the fact patches from a single experimental metapopulation may resemble themselves, due to connectivity, in the way they differ from other replicates (among-experimental metapopulation variance-covariance matrix). To account for within-metapopulation spatio-temporal dynamics, we included observation-level random effects (sensu [83]; patch $\times$ time nested in metapopulation), that were again included in a variancecovariance matrix to account for spatial correlations in demography. Because all 24 experimental metapopulations may have different internal dynamics, we estimated one within-landscape variance-covariance matrix per metapopulation.

Using the output from this Bayesian model, we calculated the posterior mean local patch variabilities, metapopulation asynchronies and metapopulation variabilities (resp. $\alpha, \beta$ and $\gamma$ variability sensu lato as described in [11]) for each treatment. $\alpha$ variability represents the temporal demographic variability at the local scale, while $\gamma$ variability is defined as the temporal variability at the metapopulation scale. We chose the multiplicative version of $\beta$ variability over the additive one as the former is described as spatial asynchrony between local patches, while the additive $\beta$ variability is calculated from $\alpha$ and multiplicative $\beta$ variability as an additional term increasing the spatial variability through asynchrony ("asynchrony-related spatial variability"). $\gamma$ variability can thus be seen as the combination between local $\alpha$ variability and multiplicative spatial $\beta$ variability.

\section{Data and code availability}

The Python code for the individual-based model is available at: https://github.com/fremorti/Metapopulation variability model; relative ODD protocol is presented as Supplementary Material SI2. The annotated R code for the Bayesian analysis of experimental data is available in Rmd and html formats at: https://github.com/mdahirel/Tetranychus-metapop$\underline{2019}$ and archived in Zenodo (doi: 10.5281/zenodo.3951429).

\section{Acknowledgments}

SM and DB were funded by FWO (Fonds voor Wetenschappelijk Onderzoek - Vlaanderen) through research grant G018017N; FM was holder of a BOF (Bijzonder Onderzoeksfonds) scholarship from Ghent University. 


\section{References}

[1] K. R. Crooks, "Relative Sensitivites of Mammalian Carnivores to Habitat Fragmentation," Conserv. Biol., vol. 16, no. 2, pp. 488-502, 2002.

[2] S. A. Cushman, "Effects of habitat loss and fragmentation on amphibians: A review and prospectus," Biol. Conserv., vol. 128, no. 2, pp. 231-240, 2006.

[3] R. A. Ims and H. P. Andreassen, "Effects of experimental habitat fragmentation and connectivity on root vole demography," J. Anim. Ecol., vol. 68, no. 5, pp. 839-852, Sep. 1999.

[4] J. L. McGuire, J. J. Lawler, B. H. McRae, T. A. Nuñez, and D. M. Theobald, "Achieving climate connectivity in a fragmented landscape," Proc. Natl. Acad. Sci., vol. 113, no. 26, pp. 7195-7200, 2016.

[5] X. Wang, F. G. Blanchet, and N. Koper, "Measuring habitat fragmentation: An evaluation of landscape pattern metrics," Methods Ecol. Evol., vol. 5, no. 7, pp. 634-646, 2014.

[6] M. G. Turner, "Landscape Ecology: The Effect of Pattern on Process," Annu. Rev. Ecol. Syst., vol. 20, no. 1, pp. 171-197, 1989.

[7] S. A. Cushman, K. McGarigal, and M. C. Neel, "Parsimony in landscape metrics: Strength, universality, and consistency," Ecol. Indic., vol. 8, no. 5, pp. 691-703, 2008.

[8] L. Tischendorf and L. Fahrig, "On the usage and measurement of landscape connectivity," Oikos, vol. 90, no. 1, pp. 7-19, 2000.

[9] M. R. Fuller, M. W. Doyle, and D. L. Strayer, "Causes and consequences of habitat fragmentation in river networks," Ann. N. Y. Acad. Sci., vol. 1355, no. 1, pp. 31-51, Oct. 2015.

[10] P. L. Thompson, B. Rayfield, and A. Gonzalez, "Loss of habitat and connectivity erodes species diversity, ecosystem functioning, and stability in metacommunity networks," Ecography (Cop.)., vol. 40, no. 1, pp. 98-108, Jan. 2017.

[11] S. Wang and M. Loreau, "Ecosystem stability in space: $\alpha, \beta$ and $\gamma$ variability," Ecol. Lett., vol. 17, no. 8, pp. 891-901, 2014.

[12] K. C. Abbott, "A dispersal-induced paradox: Synchrony and stability in stochastic metapopulations," Ecol. Lett., vol. 14, no. 11, pp. 1158-1169, 2011.

[13] S. Wang, B. Haegeman, and M. Loreau, "Dispersal and metapopulation stability," PeerJ, vol. 3, no. 10, pp. 1-16, 2015.

[14] T. C. Gouhier, F. Guichard, and A. Gonzalez, "Synchrony and Stability of Food Webs in 
Metacommunities," Am. Nat., vol. 175, no. 2, pp. E16-E34, 2010.

[15] A. L. Lloyd and R. M. May, "Synchronicity, chaos and population cycles :," Trends Ecol. Evol., vol. 14, no. 11, pp. 417-418, 1999.

[16] M. W. Sabelis, A. Janssen, O. Diekmann, V. A. A. Jansen, E. van Gool, and M. van Baalen, "Global Persistence Despite Local Extinction in Acarine Predator-Prey Systems: Lessons From Experimental and Mathematical Exercises," Adv. Ecol. Res., vol. 37, no. 04, pp. 183-220, 2005.

[17] P. J. Hudson and I. M. Cattadori, "The Moran effect: A cause of population synchrony," Trends Ecol. Evol., vol. 14, no. 1, pp. 1-2, 1999.

[18] D. Bonte et al., "Costs of dispersal," Biol. Rev., vol. 87, no. 2, pp. 290-312, May 2012.

[19] L. Govaert et al., "Eco-evolutionary feedbacks-Theoretical models and perspectives," Funct. Ecol., vol. 33, no. 1, pp. 13-30, Jan. 2019.

[20] D. Bonte, S. Masier, and F. Mortier, "Eco-evolutionary feedbacks following changes in spatial connectedness," Curr. Opin. Insect Sci., vol. 29, pp. 64-70, Oct. 2018.

[21] S. Gandon, "Kin competition, the cost of inbreeding and the evolution of dispersal," J. Theor. Biol., vol. 200, no. 4, pp. 345-364, 1999.

[22] H. J. Poethke, B. Pfenning, and T. Hovestadt, "The relative contribution of individual and kin selection to the evolution of density-dependent dispersal rates," Evol. Ecol. Res., vol. 9, no. 1, pp. 41-50, 2007.

[23] M. Dahirel, S. Masier, D. Renault, and D. Bonte, "The distinct phenotypic signatures of dispersal and stress in an arthropod model: from physiology to life history," J. Exp. Biol., vol. 222, Aug. 2019.

[24] J. Clobert, M. Baguette, T. G. Benton, and J. M. Bullock, Dispersal ecology and evolution. 2012.

[25] S. Goossens, N. Wybouw, T. Van Leeuwen, and D. Bonte, "The physiology of movement," Mov. Ecol., vol. 8, no. 5, 2020.

[26] K. H. P. Van Petegem et al., "Kin competition accelerates experimental range expansion in an arthropod herbivore," Ecol. Lett., vol. 21, no. 2, pp. 225-234, Feb. 2018.

[27] S. Masier and D. Bonte, "Spatial connectedness imposes local- and metapopulation-level selection on life history through feedbacks on demography," Ecol. Lett., vol. 23, no. 2, pp. 242-253, 2020.

[28] L. Fahrig, "Ecological Responses to Habitat Fragmentation Per Se," Annu. Rev. Ecol. Evol. Syst., vol. 48, no. 1, p. annurev-ecolsys-110316-022612, 2017.

[29] D. Bonte and Q. Bafort, "The importance and adaptive value of life-history evolution for metapopulation dynamics," J. Anim. Ecol., vol. 88, no. 1, pp. 24-34, 2019. 
[30] P. Karisto and É. Kisdi, "Joint evolution of dispersal and connectivity," Evolution (N. Y)., pp. 1-30, 2019.

[31] A. R. Ives, S. T. Woody, E. V. Nordheim, C. Nelson, and J. H. Andrews, "The Synergistic Effects of Stochasticity and Dispersal on Population Densities," Am. Nat., vol. 163, no. 3, pp. 375-387, 2004.

[32] P. W. Hedrick and A. Garcia-Dorado, "Understanding Inbreeding Depression, Purging, and Genetic Rescue," Trends Ecol. Evol., vol. 31, no. 12, pp. 940-952, 2016.

[33] E. V. Bitume et al., "Density and genetic relatedness increase dispersal distance in a subsocial organism," Ecol. Lett., vol. 16, no. 4, pp. 430-437, 2013.

[34] D. Bonte, "Inbreeding depresses short and long distance dispersal in three congeneric spiders," J. Evol. Biol., vol. 22, no. 7, pp. 1429-1434, 2009.

[35] I. Hanski and D. Simberloff, "The Metapopulation Approach, Its History, Conceptual Domain, and Application to Conservation," Metapopulation Biol., pp. 5-26, 1997.

[36] P. O. Cheptou, A. L. Hargreaves, D. Bonte, and H. Jacquemyn, "Adaptation to fragmentation: Evolutionarydynamics driven by human influences," Philosophical Transactions of the Royal Society B: Biological Sciences, vol. 372, no. 1712. Royal Society of London, 19-Jan-2017.

[37] A. De Roissart, S. Wang, and D. Bonte, "Spatial and spatiotemporal variation in metapopulation structure affects population dynamics in a passively dispersing arthropod," J. Anim. Ecol., vol. 84, no. 6, pp. 1565-1574, Nov. 2015.

[38] F. Mortier, S. Jacob, M. L. Vandegehuchte, and D. Bonte, "Habitat choice stabilizes metapopulation dynamics through increased ecological specialisation.," Oikos, vol. 128, no. 4, pp. 529-539, 2019.

[39] S. Jacob, A. S. Chaine, M. Huet, J. Clobert, and D. Legrand, "Variability in dispersal syndromes is a key driver of metapopulation dynamics in experimental microcosms," Am. Nat., vol. 194, no. 5, pp. 613-626, 2019.

[40] E. A. Fronhofer et al., "Bottom-up and top-down control of dispersal across major organismal groups: a coordinated distributed experiment," Nat. Ecol. Evol., vol. 2, no. 12, pp. 1859-1863, 2018.

[41] J. Clobert, J. F. Le Galliard, J. Cote, S. Meylan, and M. Massot, "Informed dispersal, heterogeneity in animal dispersal syndromes and the dynamics of spatially structured populations," Ecol. Lett., vol. 12, no. 3, pp. 197-209, Mar. 2009.

[42] D. Bonte and M. Dahirel, "Dispersal: a central and independent trait in life history," Oikos, vol. 126, no. 4, pp. 472-479, Apr. 2017.

[43] D. Bonte, A. De Roissart, N. Wybouw, and T. Van Leeuwen, "Fitness maximization by dispersal: Evidence from an invasion experiment," Ecology, vol. 95, no. 11, pp. 3104-3111, Nov. 2014. 
[44] P. Amarasekare and R. M. Nisbet, "Spatial heterogeneity, source-sink dynamics, and the local coexistence of competing species," Am. Nat., vol. 158, no. 6, pp. 572-584, 2001.

[45] M. Méndez, M. Vögeli, J. L. Tella, and J. A. Godoy, "Joint effects of population size and isolation on genetic erosion in fragmented populations: Finding fragmentation thresholds for management," Evol. Appl., vol. 7, no. 4, pp. 506-518, 2014.

[46] J. Pijpe, P. M. Brakefield, and B. J. Zwaan, "Increased life span in a polyphenic butterfly artificially selected for starvation resistance," Am. Nat., vol. 171, no. 1, pp. 81-90, 2008.

[47] J. Li and D. C. Margolies, "Responses to direct and indirect selection on aerial dispersal behaviour in Tetranychus urticae," Heredity (Edinb)., vol. 72, no. 1, pp. 10-22, 1994.

[48] D. A. Vasseur et al., "Increased temperature variation poses a greater risk to species than climate warming," Proc. R. Soc. B Biol. Sci., vol. 281, no. 1779, 2014.

[49] T. L. S. Vincent, D. Scheel, J. S. Brown, and T. L. Vincent, "Trade-offs and coexistence in consumer-resource models: It all depends on what and where you eat," Am. Nat., vol. 148, no. 6, pp. 1038-1058, 1996.

[50] B. K. Singh, J. S. Rao, R. Ramaswamy, and S. Sinha, "The role of heterogeneity on the spatiotemporal dynamics of host-parasite metapopulation," Ecol. Modell., vol. 180, no. 2-3, pp. 435443, 2004.

[51] R. D. Holt, "Population dynamics in two-patch environments: Some anomalous consequences of an optimal habitat distribution," Theor. Popul. Biol., vol. 28, no. 2, pp. 181-208, 1985.

[52] J. D. Van Dyken and B. Zhang, "Carrying capacity of a spatially-structured population: Disentangling the effects of dispersal, growth parameters, habitat heterogeneity and habitat clustering," J. Theor. Biol., vol. 460, pp. 115-124, 2019.

[53] D. L. Deangelis, B. Zhang, W. M. Ni, and Y. Wang, "Carrying capacity of a population diffusing in a heterogeneous environment," Mathematics, vol. 8, no. 1, 2020.

[54] S. Hamel, J.-M. Gaillard, M. Douhard, M. Festa-Bianchet, F. Pelletier, and N. G. Yoccoz, "Quantifying individual heterogeneity and its influence on life-history trajectories: Different methods for different questions and contexts," Oikos, vol. 127, no. 5, 2018.

[55] E. J. Gangloff, A. M. Sparkman, and A. M. Bronikowski, "Among-individual heterogeneity in maternal behaviour and physiology affects reproductive allocation and offspring life-history traits in the garter snake Thamnophis elegans," Oikos, vol. 127, no. 5, pp. 612-625, 2018.

[56] I. M. Smallegange, R. E. Fernandes, and J. C. Croll, "Population consequences of individual heterogeneity in life histories: overcompensation in response to harvesting of alternative reproductive tactics," Oikos, vol. 127, no. 5, pp. 738-749, 2018. 
[57] Y. Vindenes, S. Engen, and B. E. Sæther, "Individual heterogeneity in vital demographic stochasticity," Am. Nat., vol. 171, no. 4, pp. 455-467, 2008.

[58] S. Wang, M. Loreau, D. Platz, and M. Loreau, "Biodiversity and ecosystem stability across scales in metacommunities," Ecol. Lett., vol. 19, no. 5, pp. 510-518, May 2016.

[59] M. A. Strodl and P. Schausberger, "Social familiarity relaxes the constraints of limited attention and enhances reproduction of group-living predatory mites," Oikos, vol. 122, no. 8, pp. 1217-1226, 2013.

[60] K. Oku, S. Magalhães, and M. Dicke, "The presence of webbing affects the oviposition rate of twospotted spider mites, Tetranychus urticae (Acari: Tetranychidae)," Exp. Appl. Acarol., vol. 49, no. 3, pp. 167-172, 2009.

[61] S. Yano, "Cooperative web sharing against predators promotes group living in spider mites," Behav. Ecol. Sociobiol., vol. 66, no. 6, pp. 845-853, 2012.

[62] J. Monro, "The Exploitation and Conservation of Resources by Populations of Insects," J. Anim. Ecol., vol. 36, no. 3, pp. 531-547, 1967.

[63] J. A. Wiens, "Population Responses to Patchy Environments," Annu. Rev. Ecol. Syst., vol. 7, no. 1, pp. 81-120, 1976.

[64] S. Aucejo-Romero, A. Gómez-Cadenas, and J. A. Jacas-Miret, "Effects of NaCl-stressed citrus plants on life-history parameters of Tetranychus urticae (Acari: Tetranychidae)," Exp. Appl. Acarol., vol. 33, no. 1-2, pp. 55-67, 2004.

[65] L. Fahrig, "Effect of Habitat Fragmentation on the Extinction Threshold: A Synthesis," Ecol. Appl., vol. 12, no. 2, pp. 346-353, Apr. 2002.

[66] C. J. Briggs and M. F. Hoopes, "Stabilizing effects in spatial parasitoid-host and predator-prey models: A review," Theor. Popul. Biol., vol. 65, no. 3, pp. 299-315, 2004.

[67] R. Lande, S. Engen, and S. Bernt-Erik, "Extinction Times in Finite Metapopulation Models with Stochastic Local Dynamics," Oikos, vol. 83, no. 2, pp. 383-389, 1998.

[68] T. Vogwill, A. Fenton, A. Buckling, M. E. Hochberg, and M. A. Brockhurst, "Source Populations Act as Coevolutionary Pacemakers in Experimental Selection Mosaics Containing Hotspots and Coldspots," Am. Nat., vol. 173, no. 5, pp. E171-E176, 2009.

[69] A. R. Weeks et al., "Assessing the benefits and risks of translocations in changing environments: A genetic perspective," Evol. Appl., vol. 4, no. 6, pp. 709-725, 2011.

[70] D. I. Bolnick et al., "Why intraspecific trait variation matters in community ecology," Trends Ecol. Evol., vol. 26, no. 4, pp. 183-192, 2011. 
[71] B. H. McRae, S. A. Hall, P. Beier, and D. M. Theobald, "Where to Restore Ecological Connectivity? Detecting Barriers and Quantifying Restoration Benefits," PLoS One, vol. 7, no. 12, 2012.

[72] J. M. Bullock et al., "Human-Mediated Dispersal and the Rewiring of Spatial Networks," Trends Ecol. Evol., vol. 33, no. 12, pp. 958-970, 2018.

[73] D. A. Rudnick et al., "The role of landscape connectivity in planning and implementing conservation and restoration priorities," Issues Ecol., vol. 13, no. 16, pp. 1-23, 2012.

[74] S. P. Brady et al., "Understanding maladaptation by uniting ecological and evolutionary perspectives," Am. Nat., vol. 194, no. 4, pp. 495-515, 2019.

[75] S. P. Brady et al., "Causes of maladaptation," Evol. Appl., vol. 12, no. 7, pp. 1229-1242, 2019.

[76] J. M. J. Travis and C. Dytham, "The evolution of dispersal in a metapopulation: A spatially explicit, individual-based model," Proc. R. Soc. B Biol. Sci., vol. 265, no. 1390, pp. 17-23, 1998.

[77] M. Goltsman, E. P. Kruchenkova, S. Sergeev, P. J. Johnson, and D. W. Macdonald, "Effects of food availability on dispersal and cub sex ratio in the Mednyi Arctic fox," Behav. Ecol. Sociobiol., vol. 59, no. 2, pp. 198-206, 2005.

[78] V. Baglione, D. Canestrari, J. M. Marcos, and J. Ekman, "Experimentally increased food resources in the natal territory promote offspring philopatry and helping in cooperatively breeding carrion crows," Proc. R. Soc. B Biol. Sci., vol. 273, no. 1593, pp. 1529-1535, 2006.

[79] M. Grbic et al., "Mity model: Tetranychus urticae, a candidate for chelicerate model organism," BioEssays, vol. 29, no. 5, pp. 489-496, May 2007.

[80] T. Hance and G. Van Impe, "The influence of initial age structure on predator - prey interaction," Ecol. Modell., vol. 114, pp. 195-211, 1999.

[81] T. Van Leeuwen et al., "Mitochondrial heteroplasmy and the evolution of insecticide resistance: nonMendelian inheritance in action.," Proc. Natl. Acad. Sci. U. S. A., vol. 105, no. 16, pp. 5980-5985, Apr. 2008.

[82] M. Cazaux et al., "Application of Two-spotted Spider Mite \&lt;em\&gt;Tetranychus urticae\&lt;/em\&gt; for Plant-pest Interaction Studies," J. Vis. Exp., no. 89, pp. 1-7, 2014.

[83] X. A. Harrison, "Using observation-level randomeffects to model overdispersion in count data in ecology and evolution," PeerJ, vol. 2014, no. 1, 2014. 
Figures and Tables

Figure 1. Comparison between IBM results (in each block, left) and real-life data (right) for local variability, synchronicity and global variability. Variabilities are plotted against (from left): mortality during dispersal (from high to low), global fragmentation (from low to high), dispersal frequency (from high to low) and dispersal timing (from early to late).
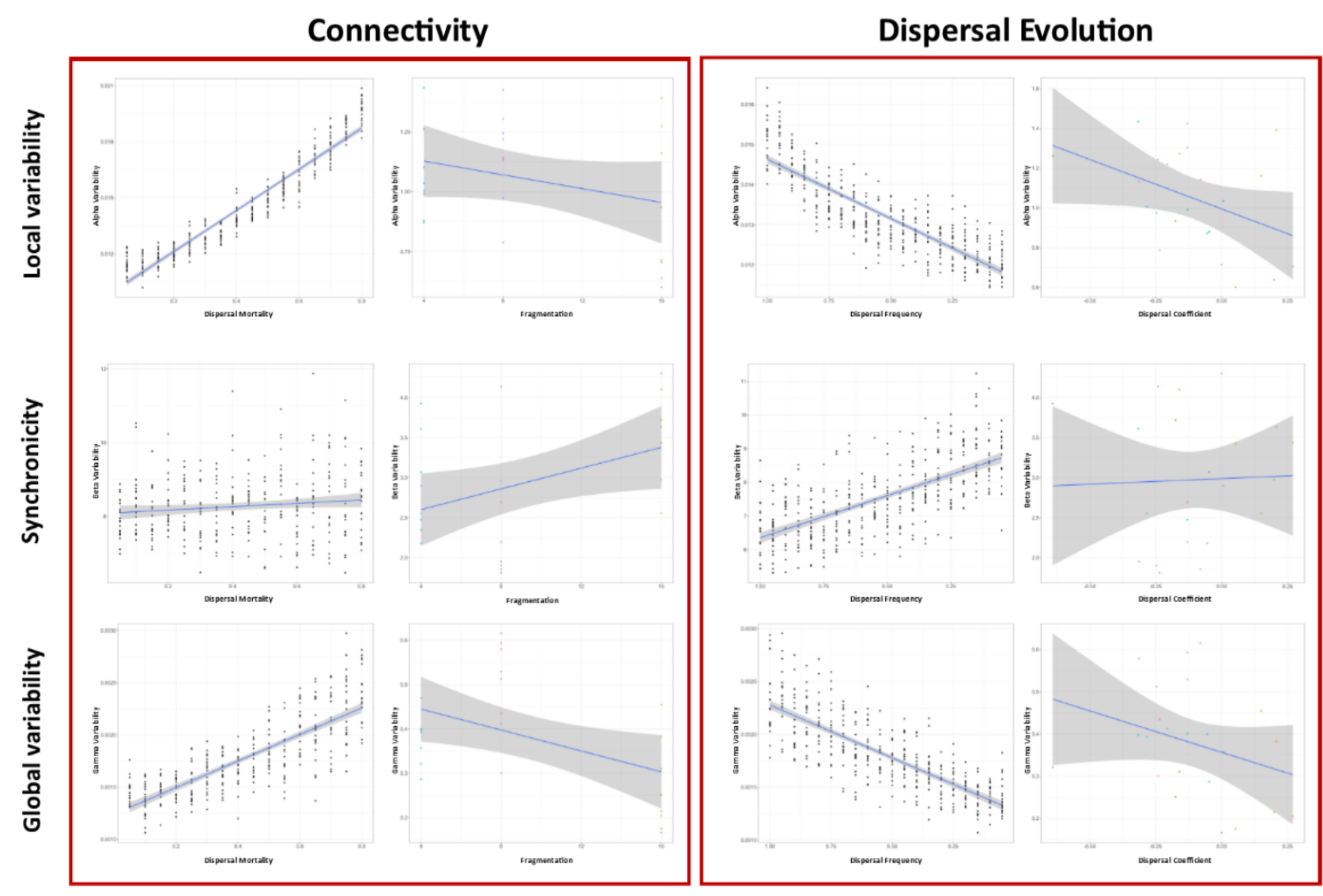
Figure 2: posterior distributions of average metapopulation density $(A), \alpha(B), \beta$ $(C)$ and $y$ variability $(D)$ ( $B$ and $D$ only plotted up to $\approx 99$ th quantile for readability) depending on global connectedness (length of the connections between patches) when no randomization (2.1) or randomization (2.2) was applied. Dots indicate posterior means, horizontal bars 66 and $95 \%$ credible intervals.

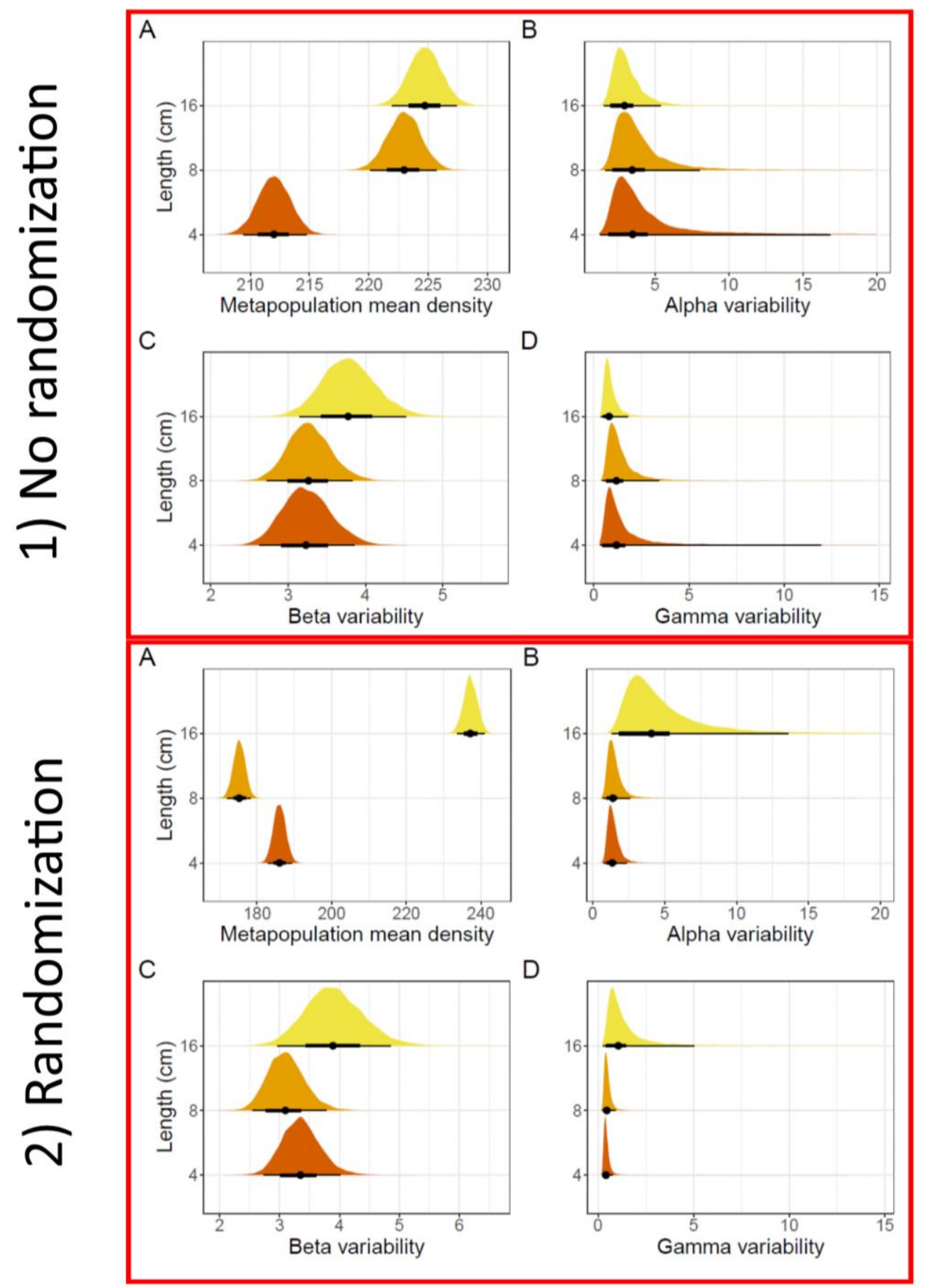


Figure 3: Mean effect of randomization on average metapopulation density $(A), \alpha$ $(B), \beta(C)$ and $y$ variability $(D)$ depending on global connectedness. Posterior distributions of the ratios mean randomized $_{\text {/mean }}$ control are plotted for each variable of interest ( $B$ and $D$ only plotted up to $\approx 99$ th quantile for readability). Dots indicate posterior means, horizontal bars 66 and 95\% credible intervals.

A

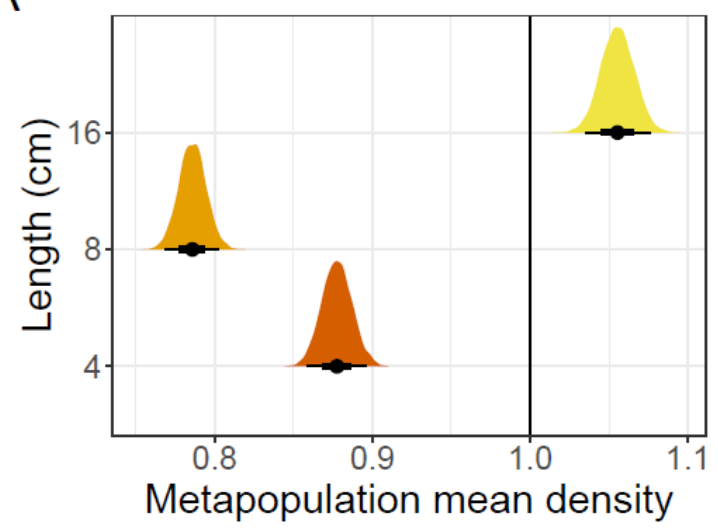

C

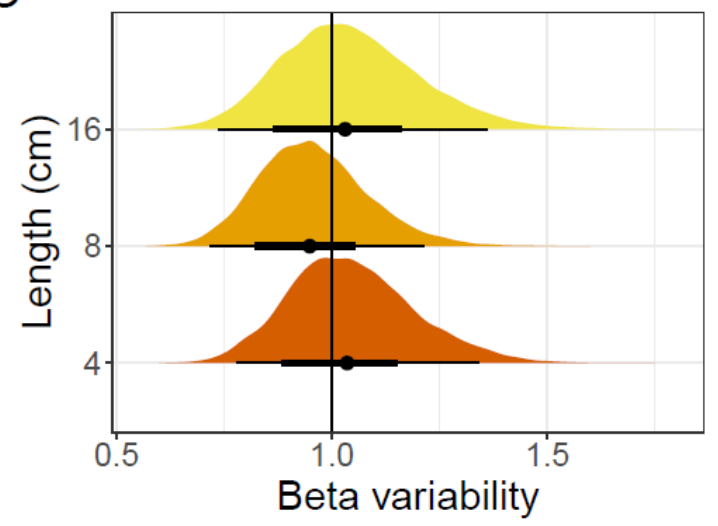

$\mathrm{B}$

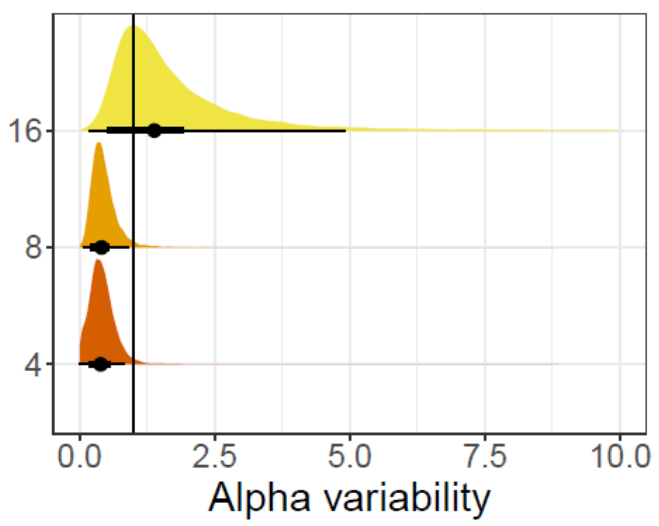

$\mathrm{D}$

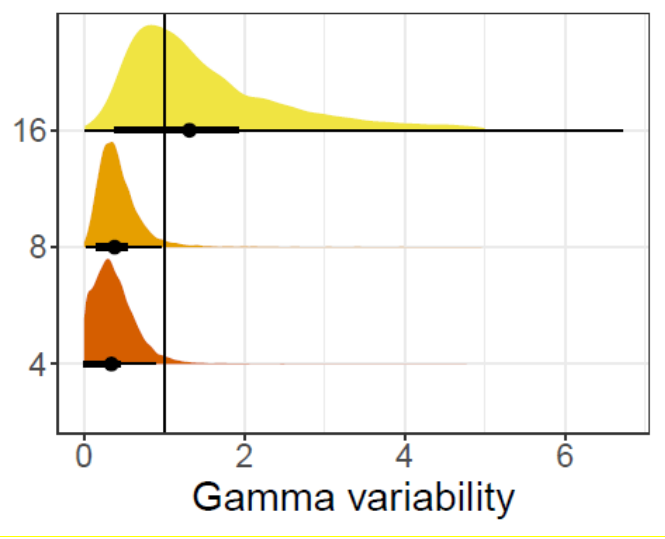


Figure 4: posterior distributions of average local population sizes of differently connected patches (central, corner or lateral), depending on global connectedness and randomization treatment. Dots indicate posterior means, horizontal bars 66 and 95\% credible intervals.

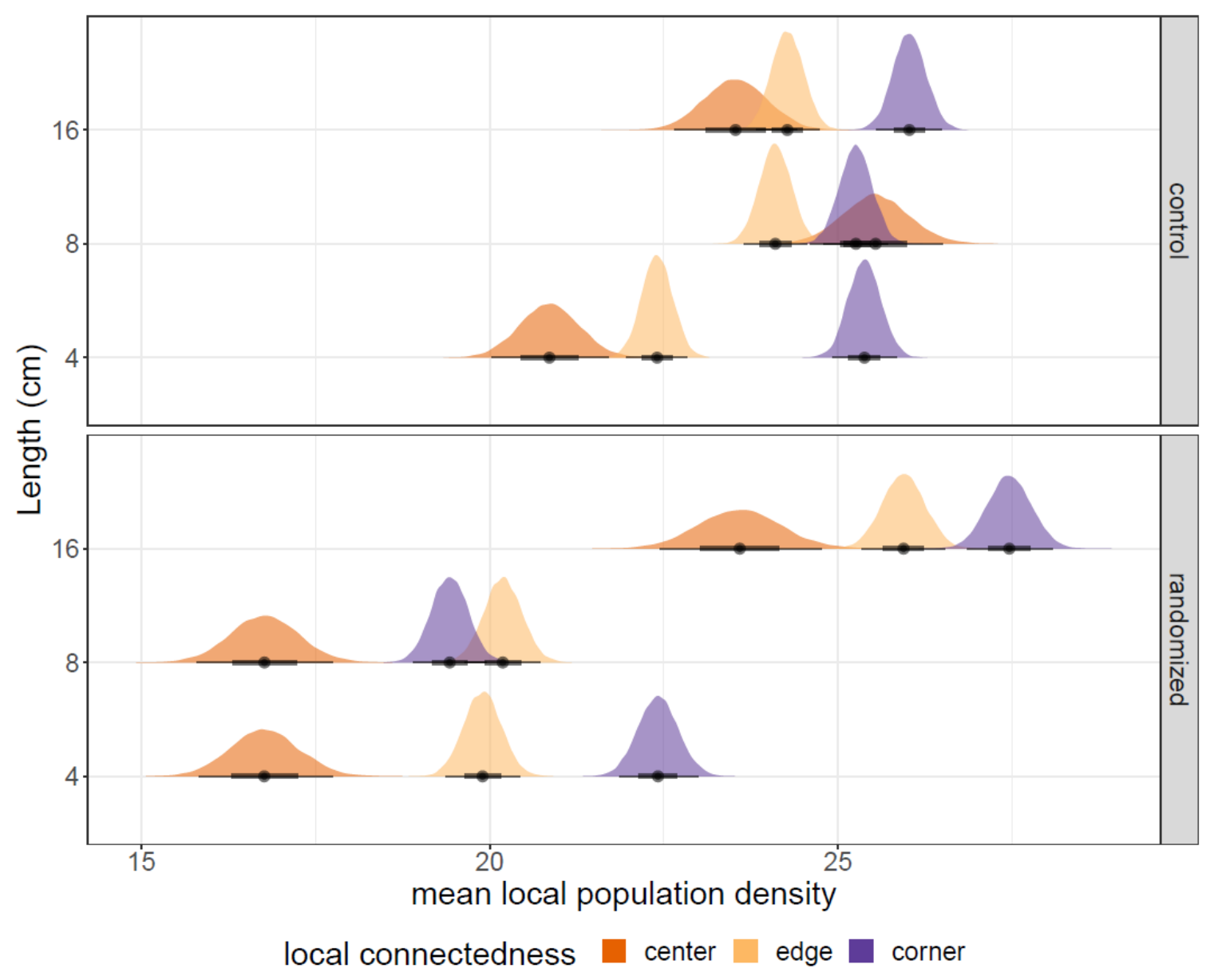


Connectivity

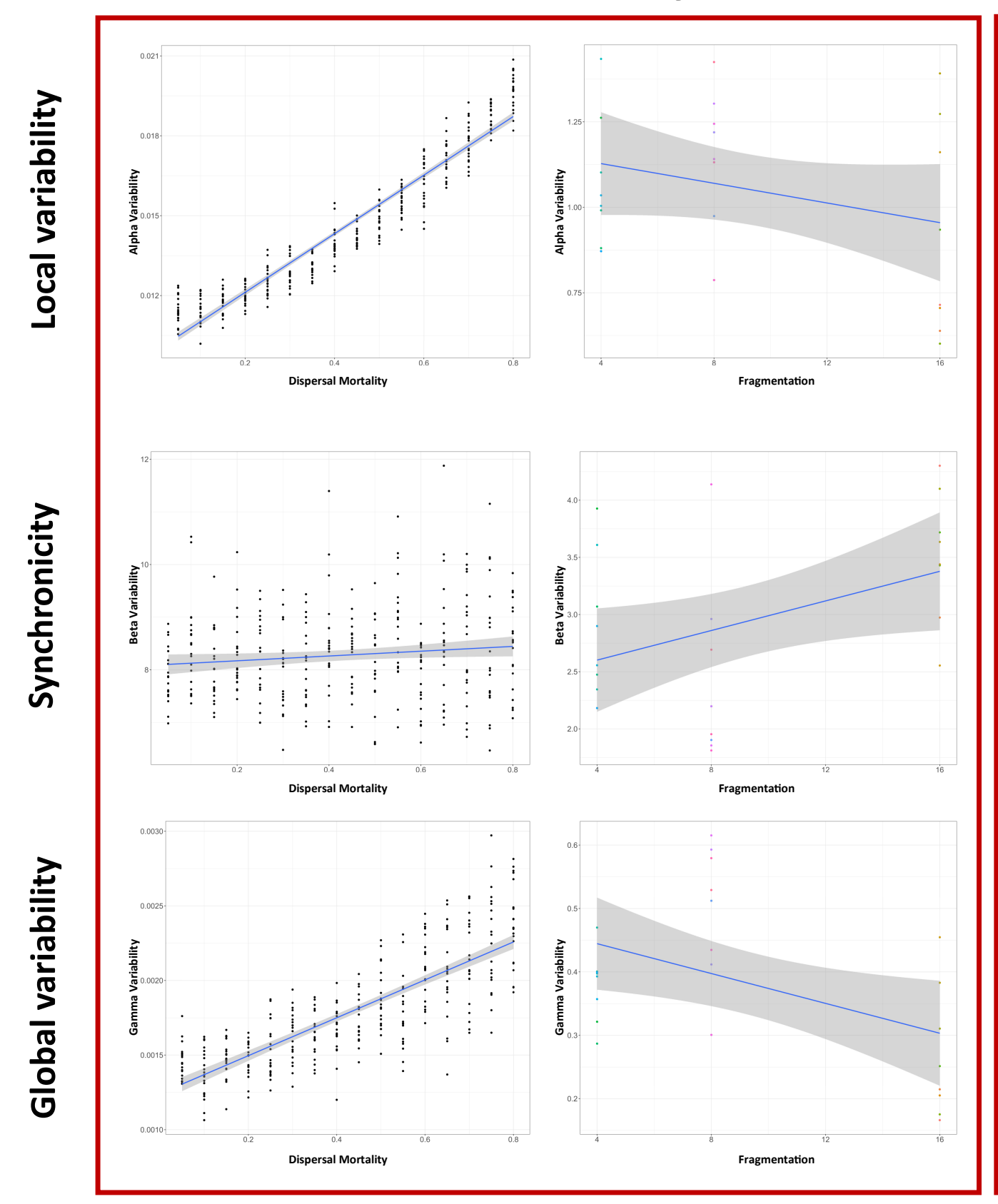

Dispersal Evolution

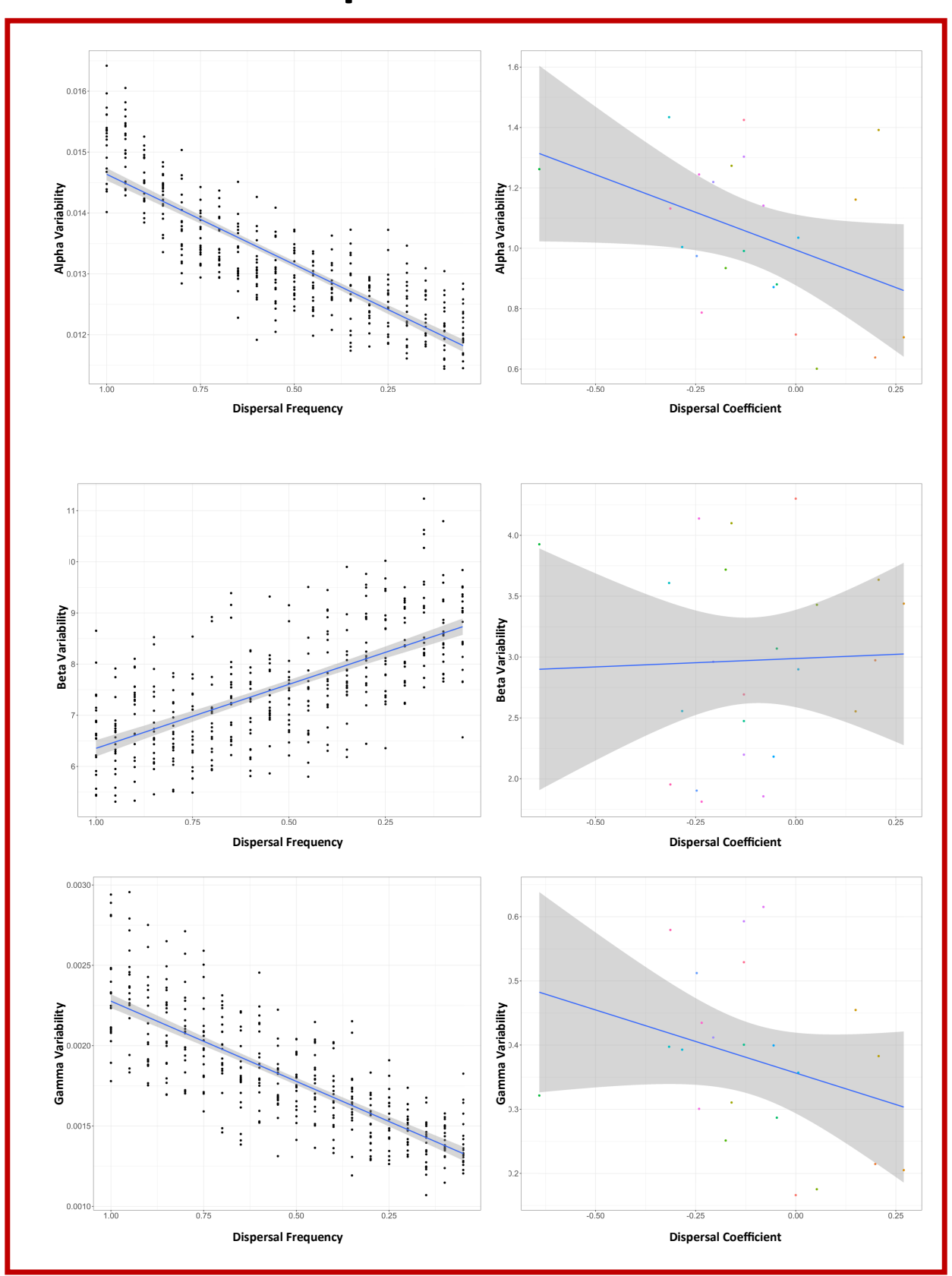


A

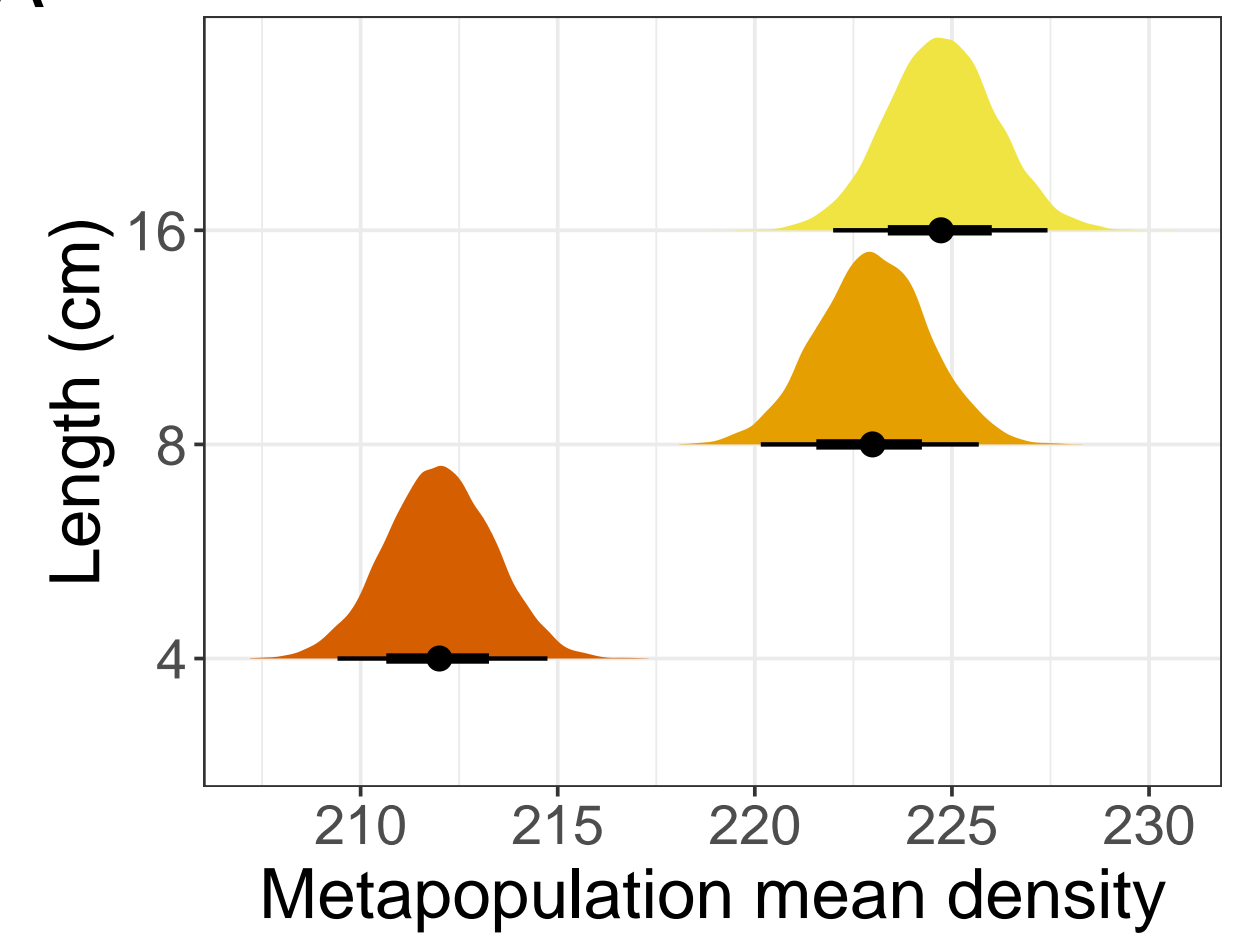

C

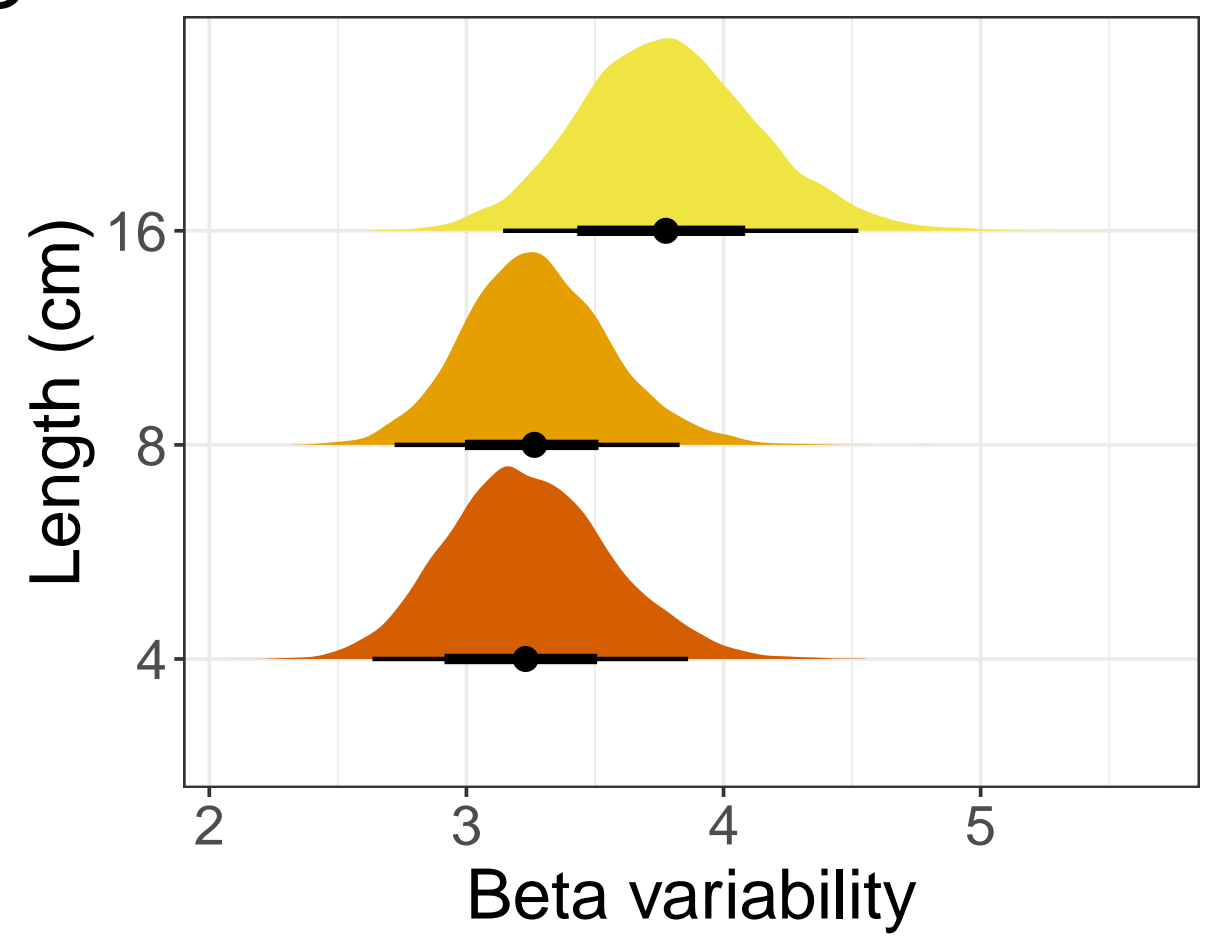

B

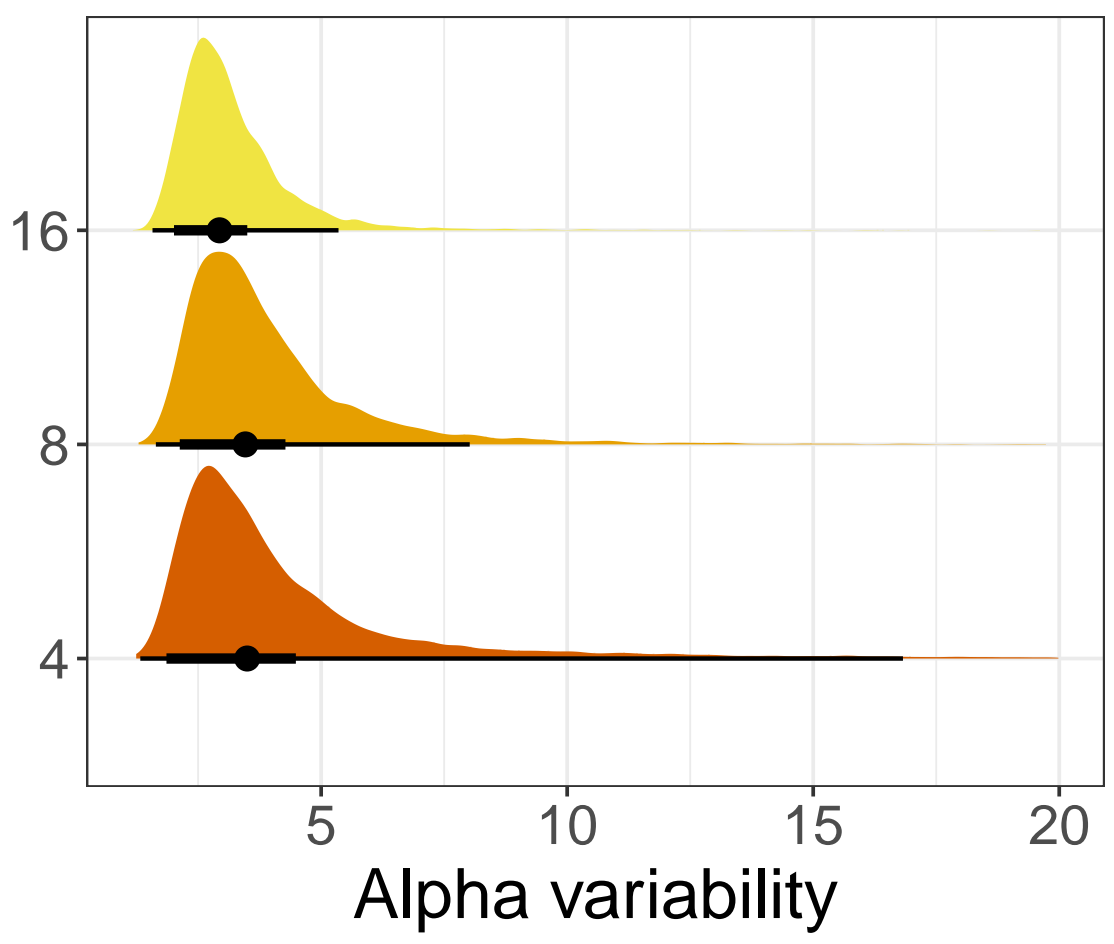

D

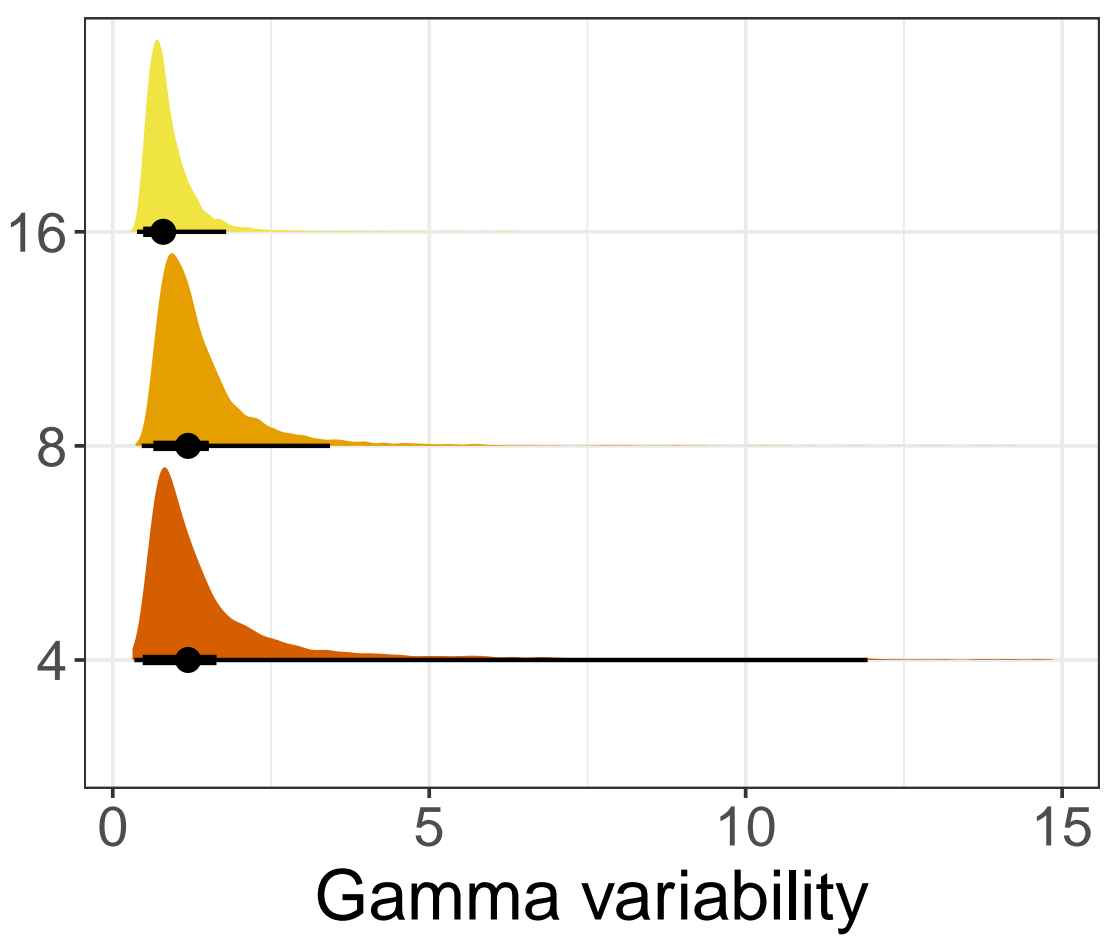


A

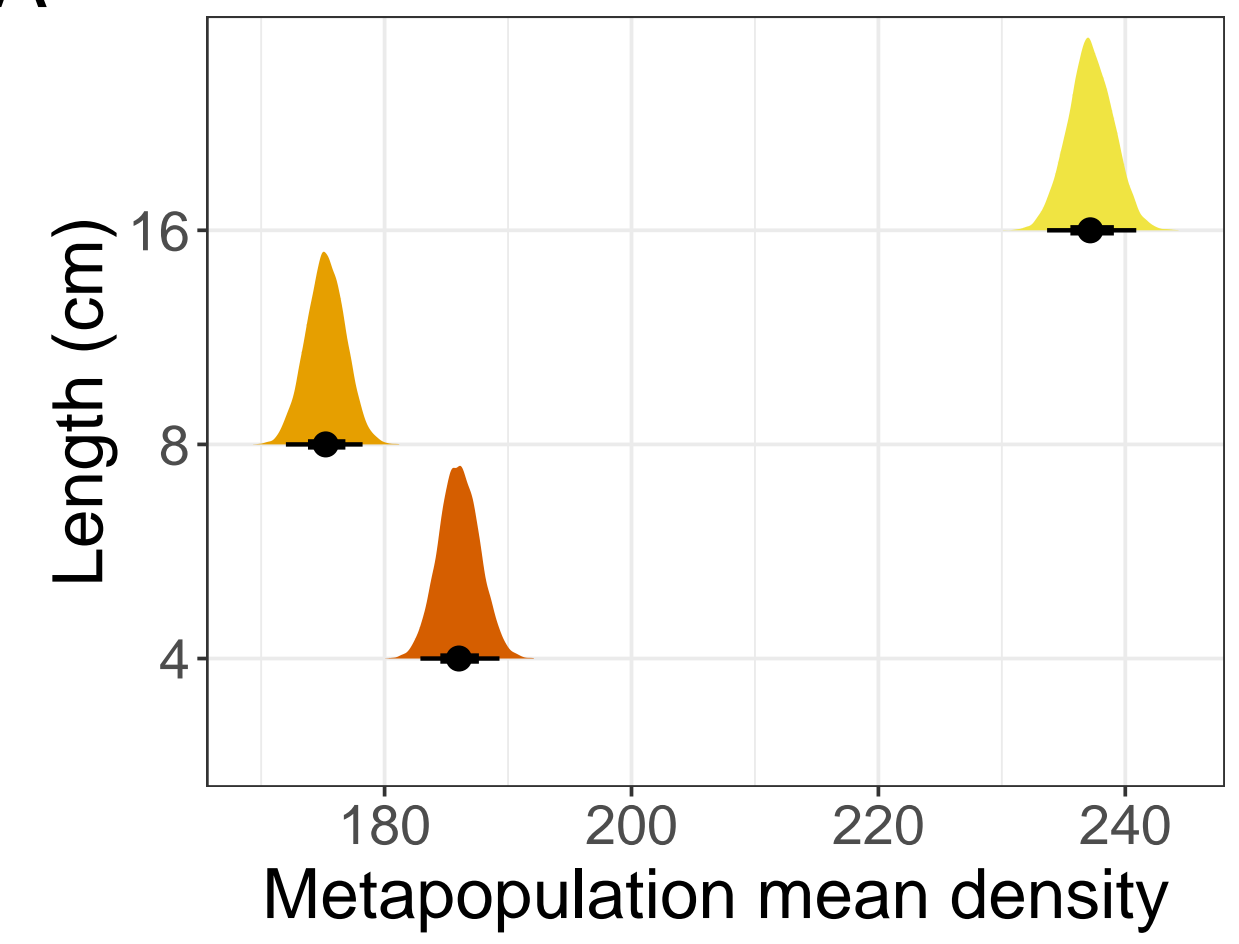

C

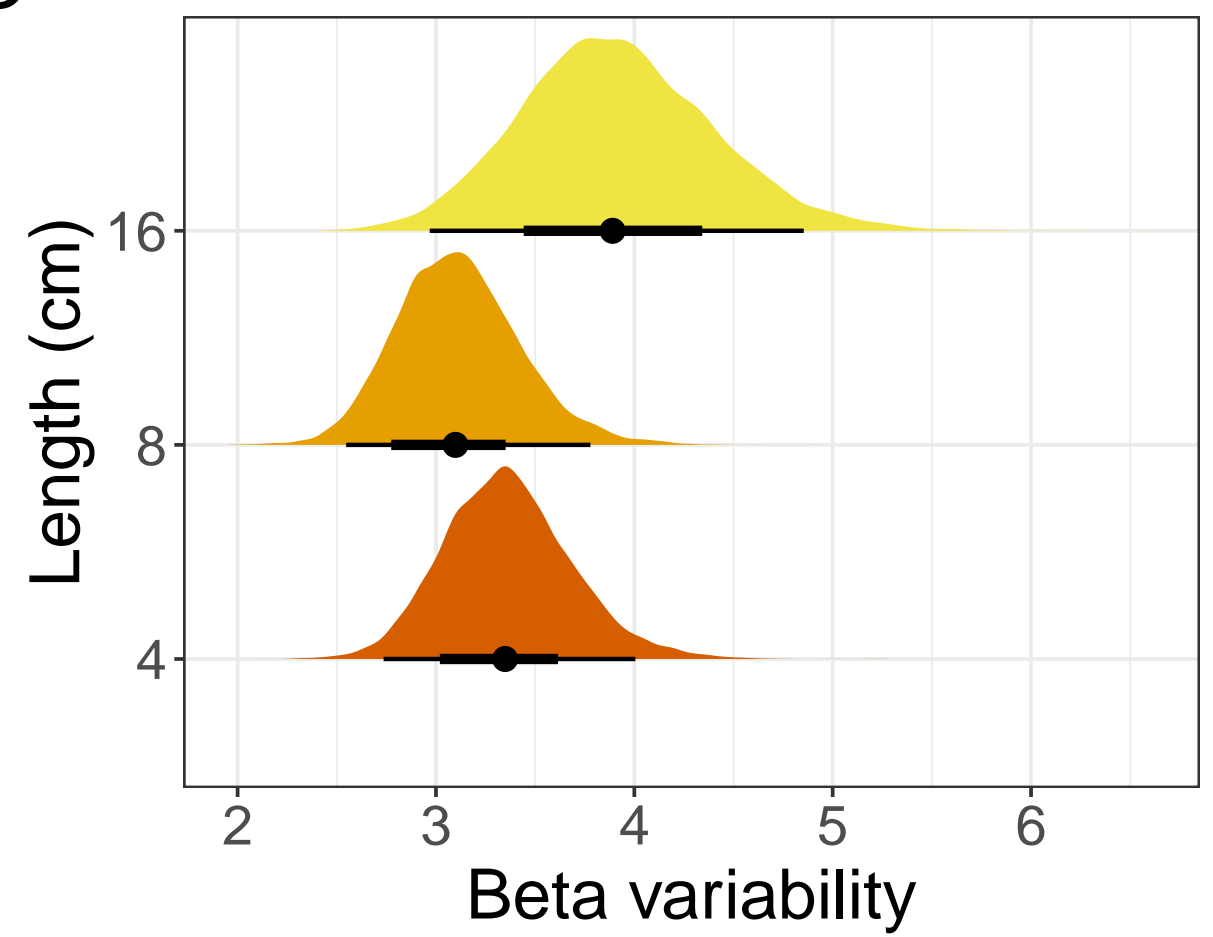

B

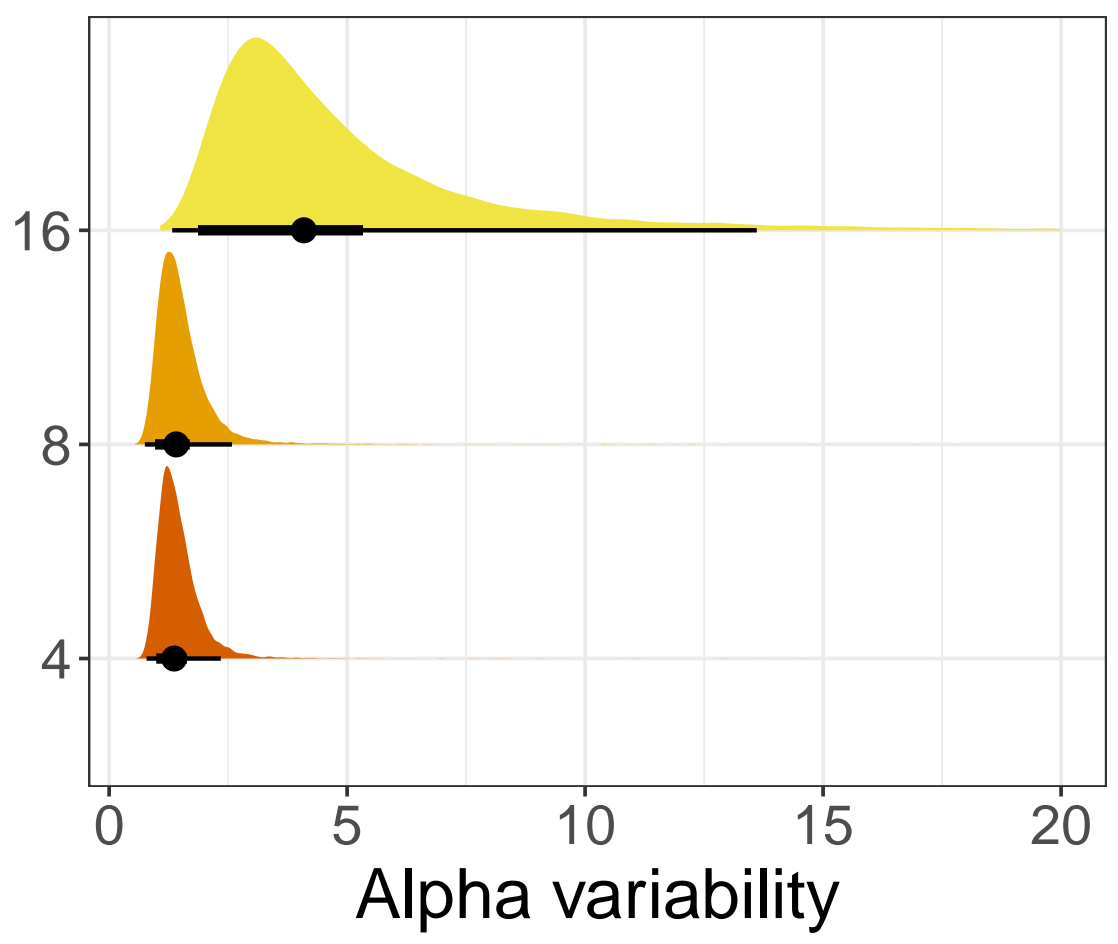

D

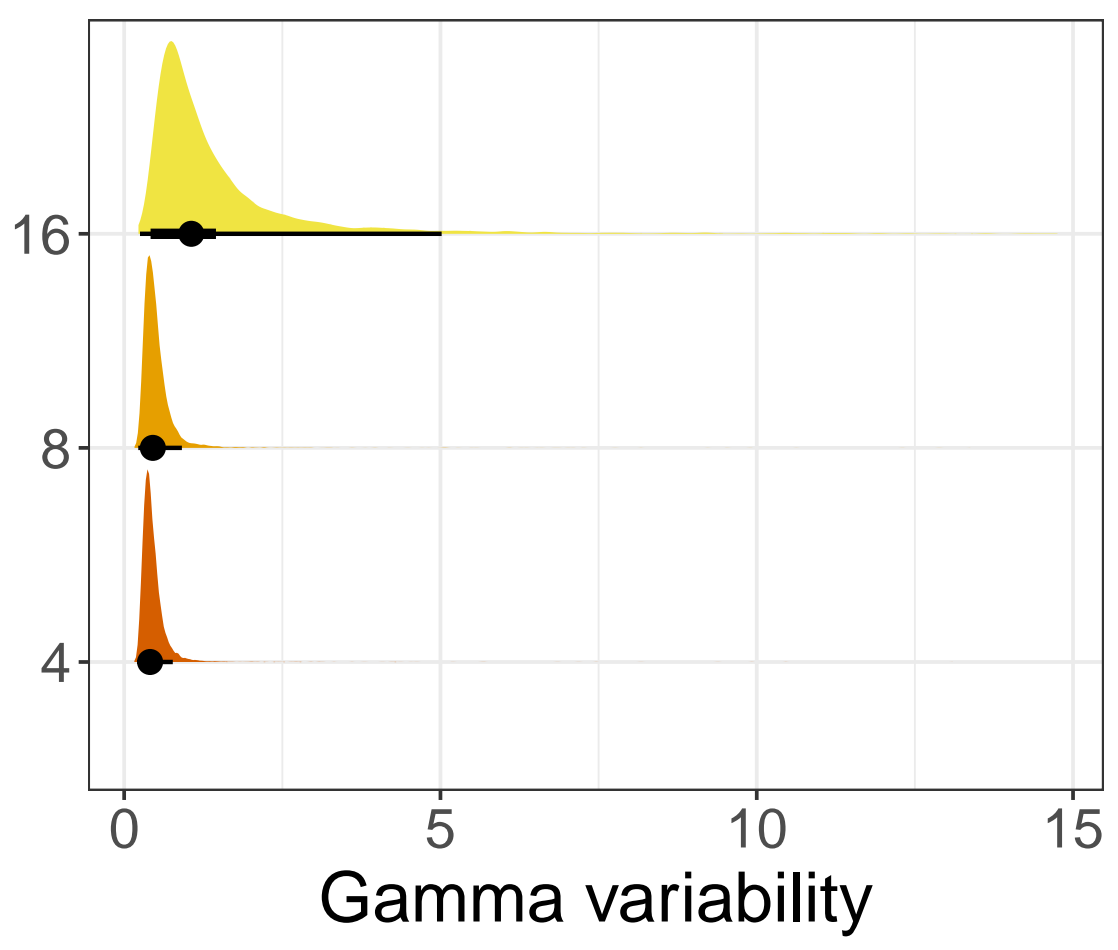


Comparisons, ratio shuffled / control

A

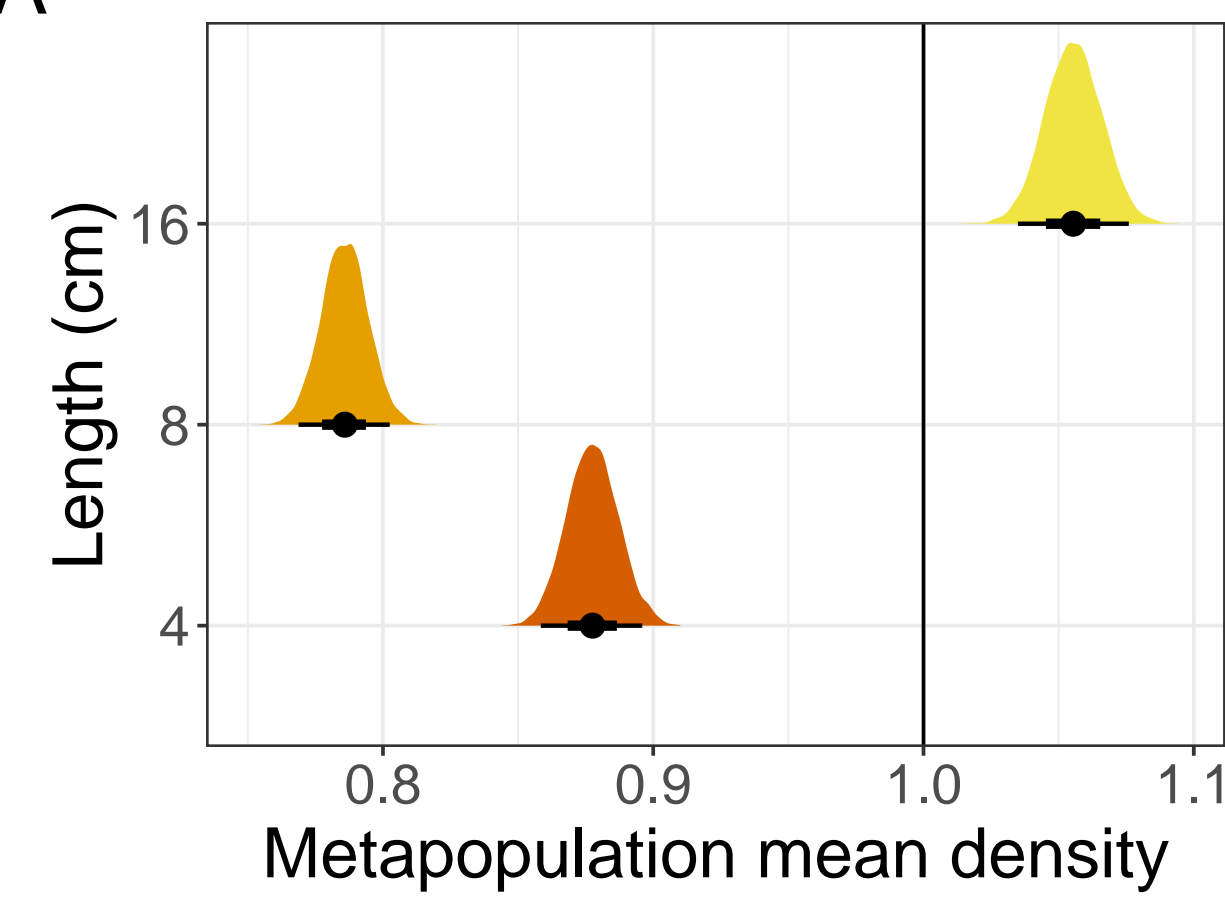

C

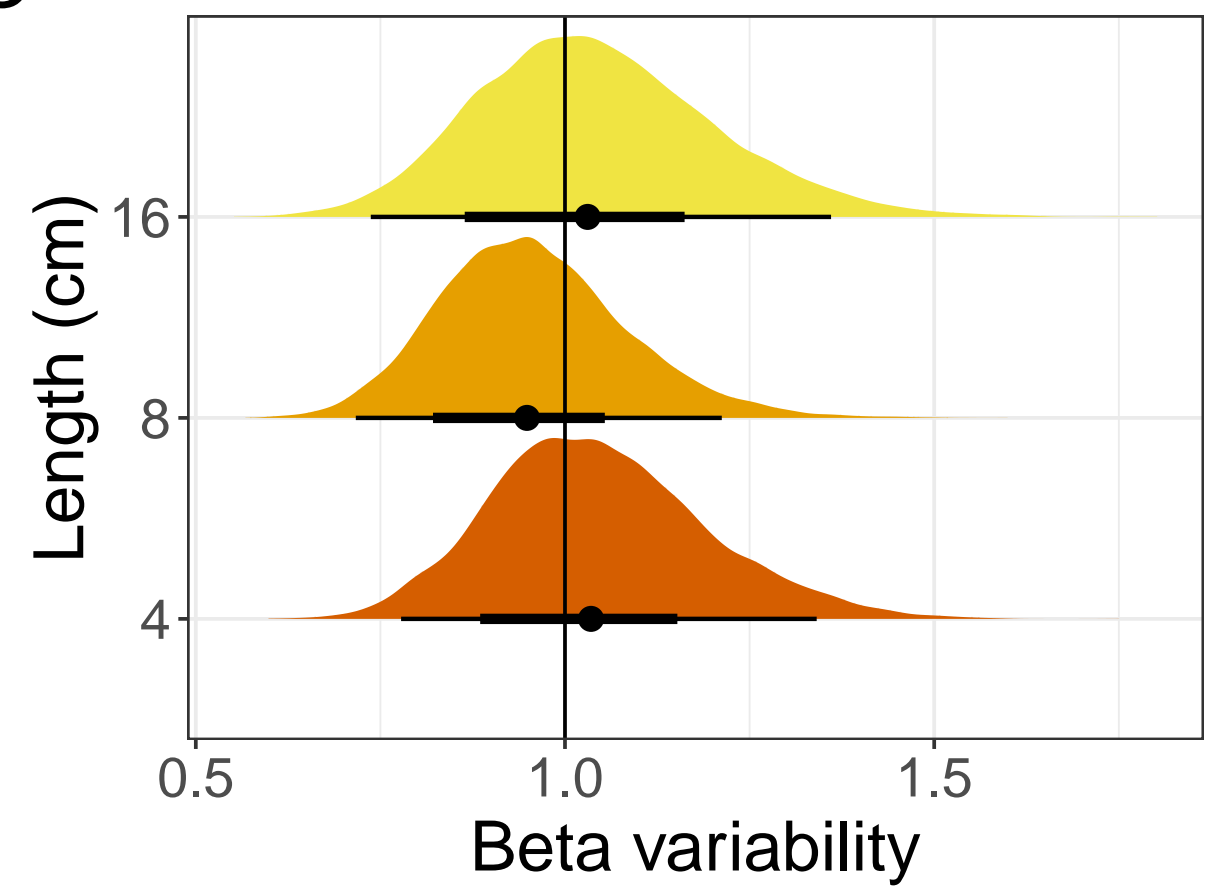

B

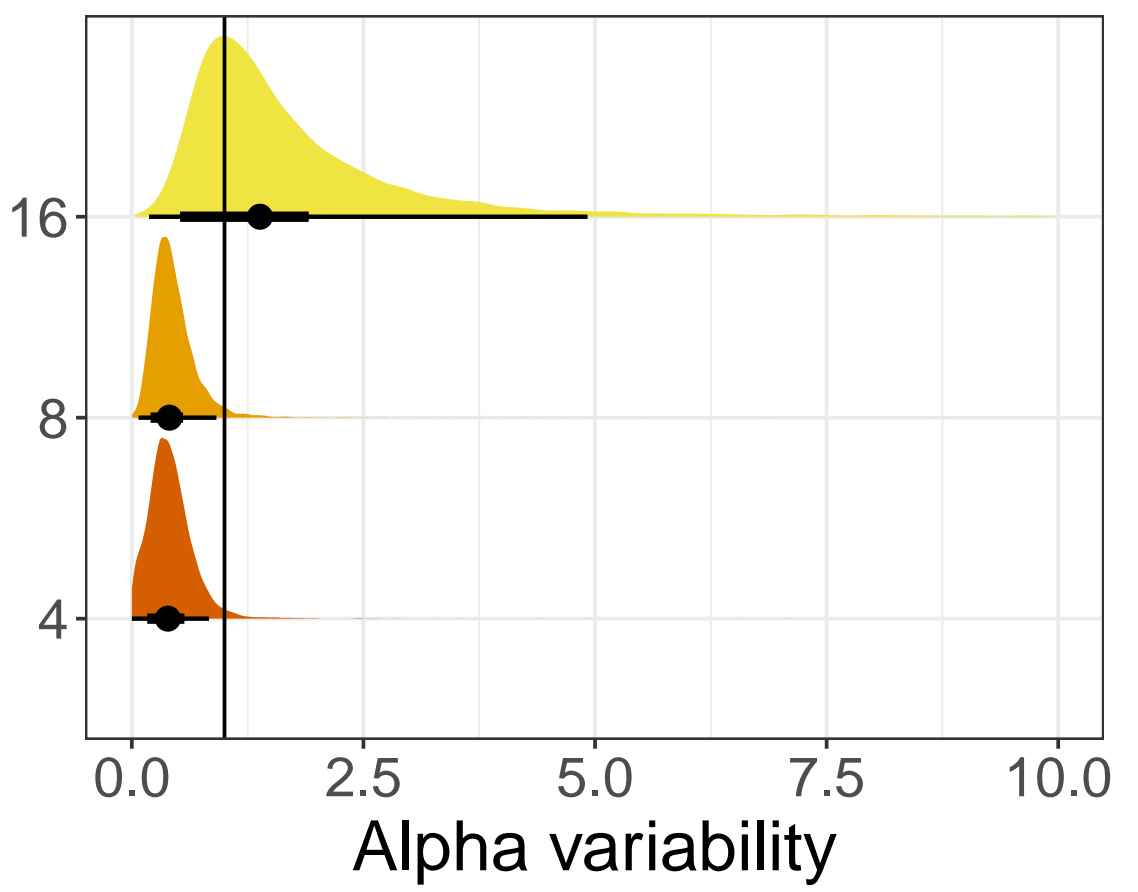

D

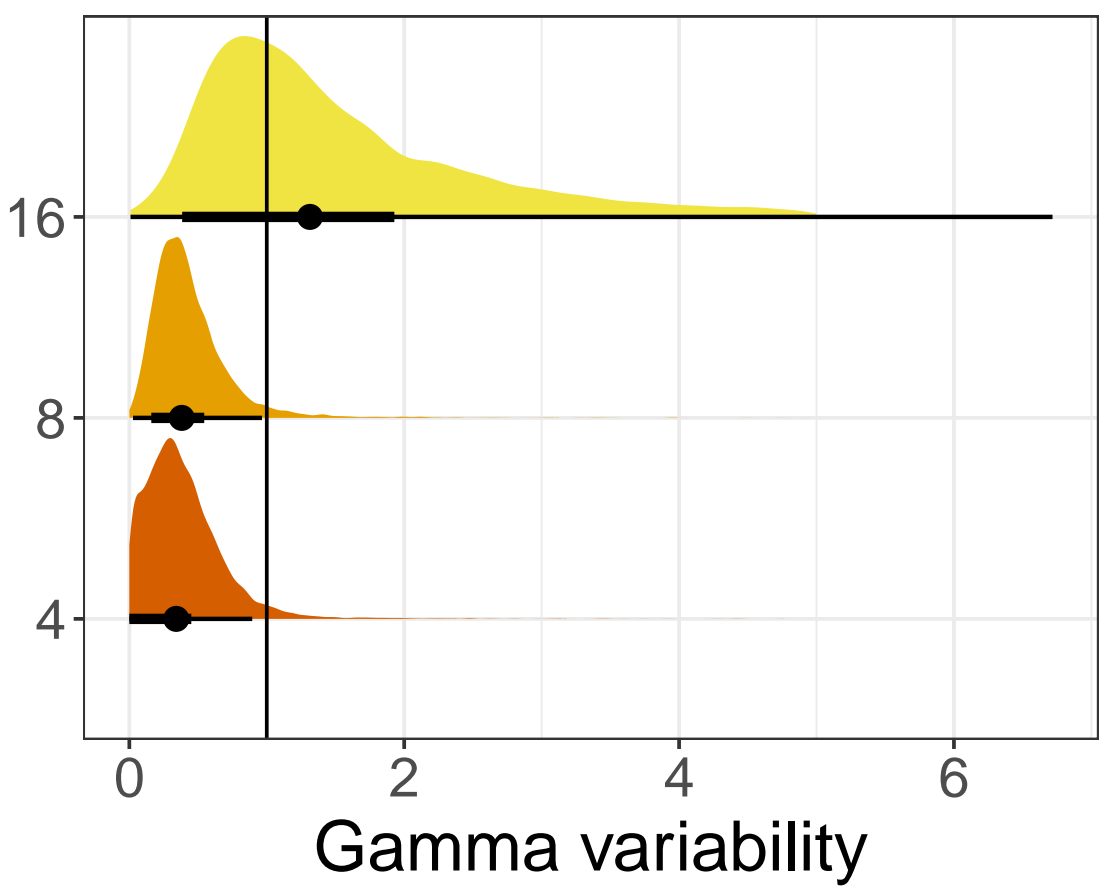




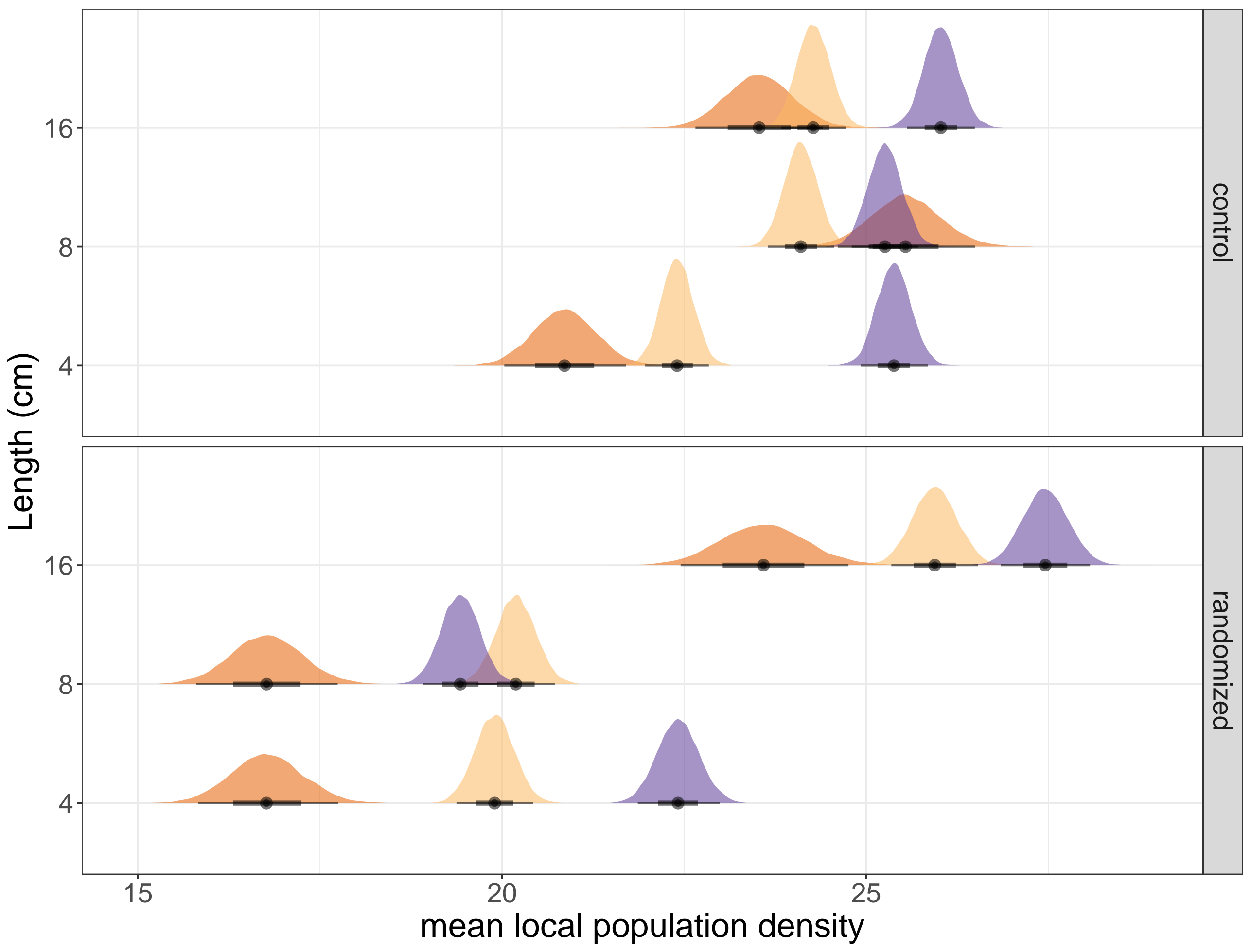

local connectedness center $\square$ edge $\square$ corner 